
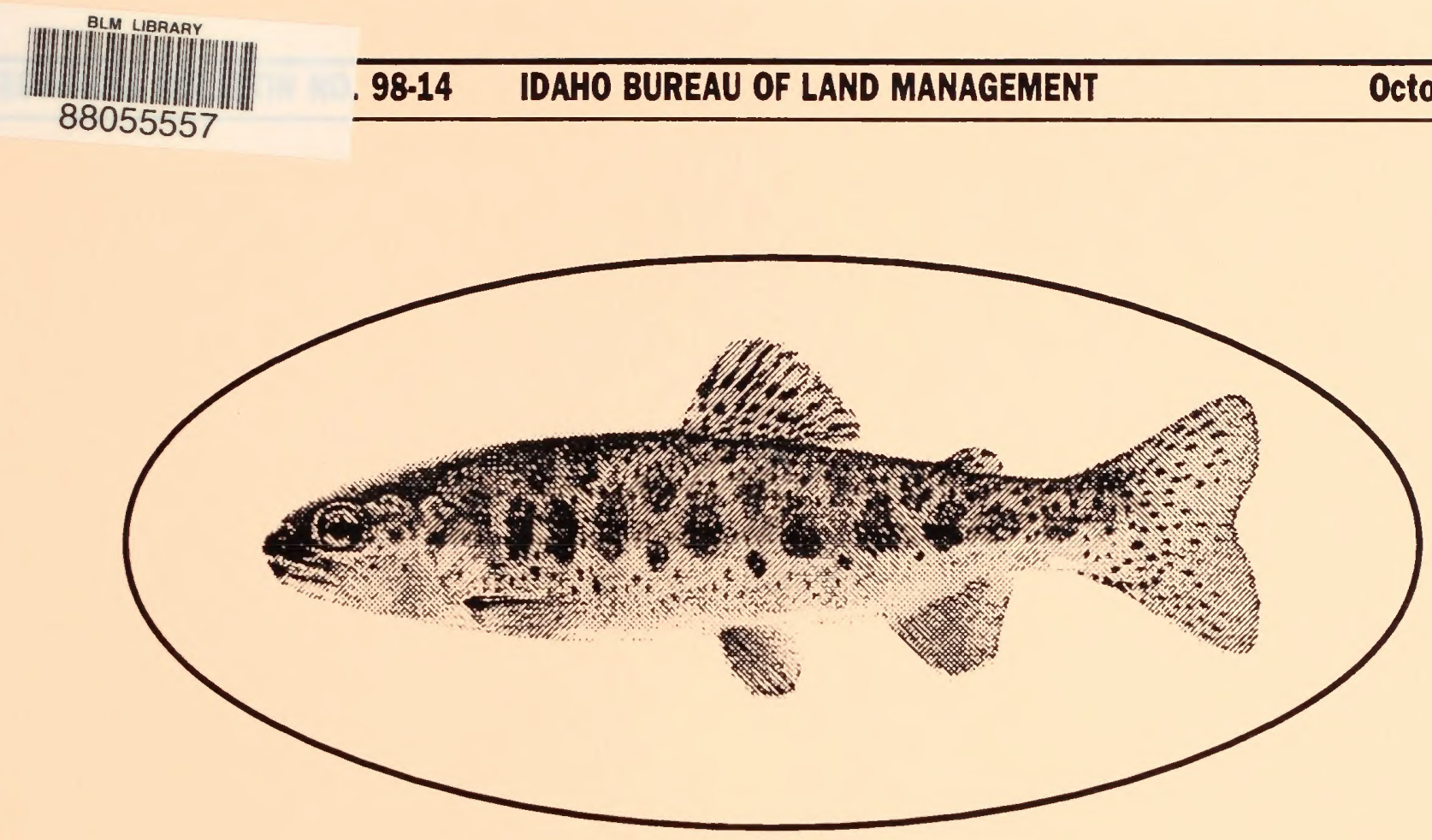

\title{
Redband Trout Oncorhynchus mykiss gairdneri Population and Stream Habitat Surveys in Northern Owyhee County and the Owyhee River and Its Tributaries, 1997
}

\author{
by \\ Dale B. Allen, \\ Brian J. Flatter, \\ Jon Nelson, \\ and Chris Medrow
}

QL

84.2

. $\mathrm{L} 352$

no.

$98-14$ 



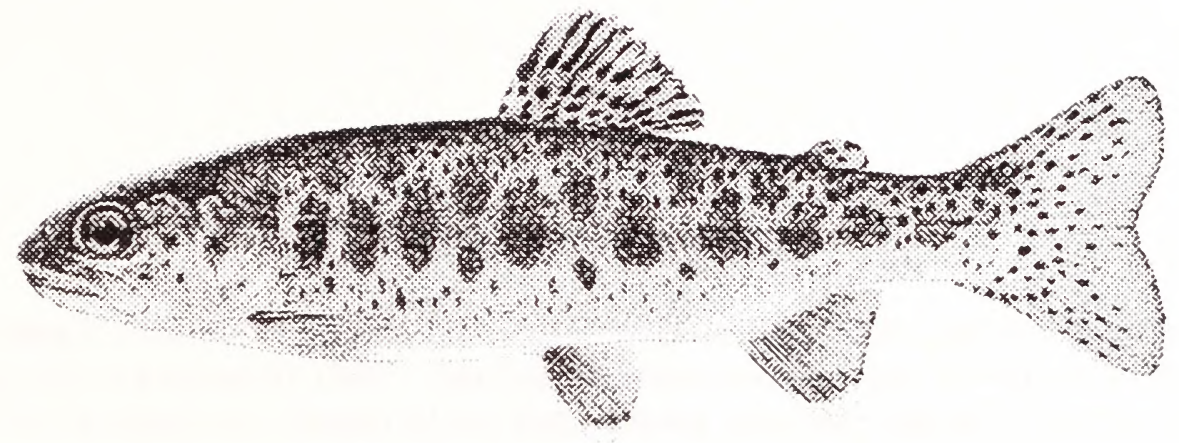

\title{
REDBAND TROUT Oncorhynchus mykiss gairdneri POPULATION AND STREAM HABITAT SURVEYS IN NORTHERN OWYHEE COUNTY AND THE OWYHEE RIVER AND ITS TRIBUTARIES, 1997
}

\author{
Prepared for \\ United States Department of the Interior \\ Bureau of Land Management \\ Lower Snake River District \\ Boise Field Office \\ 3948 Development Avenue \\ Boise, Idaho 83705-5389
}

By

Dale B. Allen,

Brian J. Flatter,

Jon Nelson, and Chris Medrow

Idaho Department of Fish \& Game

3101 South Powerline Road

Nampa, Idaho 83686

June, 1998 



\section{ABSTRACT}

Redband trout Oncorhynchus mykiss gairdneri were collected in ten of seventeen stream segment sampled in 1997. Densities of redbands ranged from 0 to $31.0 / 100 \mathrm{M}^{2}$ for all sizes of trout collected. Seven of the sites visited had been sampled since 1993 in this study, and generally trout densities had increased. The sample sites were located on Jordan Creek, Flint Creek, Reynolds Creek, Macks Creek, Salmon Creek, Squaw Creek, Sinker Creek, Scotch Bob Creek, Deep Creek, Red Canyon Creek, and the North Fork Owyhee River. The Owyhee River was sampled by angling from the Duck Valley Indian Reservation to the Oregon border by utilizing small inflatable rafts, few redband trout were captured.

Habitat information was collected on most stream segments sampled.

Water quality measurements of temperature, $\mathrm{pH}$, conductivity, and hardness were taken and values were acceptable for trout survival. Eight recording thermographs were placed into stream segments that were sampled for redband densities. Two of the thermographs recorded water temperatures consistently greater than $25 \mathrm{C}$. One of these streams had a redband population the other did not. 



\section{INTRODUCTION}

This report presents redband trout Oncorhynchus mykiss gairdneri population and stream habitat data collected in 1997 on the Bruneau and Owyhee Resource Areas of the Bureau of Land Management (BLM) lands in Owyhee County, Idaho. Data was collected by Idaho Department of Fish and Game (IDFG) Southwest Region fisheries management staff in a cooperative project with the Lower Snake River District, BLM. This report documents the fifth field season of stream and habitat surveys conducted by IDFG. Previous survey data were reported in Allen et al., 1994, 1995, 1997a and 1997b.

Redband trout historically occupied perennial drainages in Owyhee County, Idaho (Behnke, 1992). Sampling of these redband trout populations by BLM staff from 19761991 documented fragmented populations composed of small numbers of redband trout. Drought conditions experienced from 1987-1994 likely negatively impacted these redband trout populations. Unfortunately, accurate distribution maps documenting the presence or absence of redband trout in Owyhee County streams were not available to help document changes in redband distributions. The objectives of this investigation were:

(1) To determine redband trout density estimates for previously sampled stream segments

(2) To establish trout density estimates for unsurveyed stream segments

(3) To measure stream habitat variables and water quality

(4) To revisit sites sampled in 1993 to document trout population responses following "normal" water years after the drought

\section{STUDY AREA}

Seven of the seventeen sites sampled in 1997 had been sampled within the last four years, one was a historical site, and the remaining sites were all new in 1997 (Table 1). The nine new sites lie in drainages connected to the Snake River on the northern edge of Owyhee County. A float trip was taken on the Owyhee River in early July 1997 to survey the inaccessible mouths of the major tributaries to the Owyhee River for redband trout populations. 



\section{METHODS}

\section{Fish Populations}

The 1997 sample sites were chosen to document trout populations in northwestern Owyhee County, and along the Owyhee River. Seven sites that had been sampled previously by IDFG were revisited to document any trout density changes.

Sample stream segments were approximately $61 \mathrm{~m}$ in length. The upstream and downstream sample segment boundaries were located at stream constrictions to minimize fish migration during electrofishing.

A Smith-Root Model 15-B backpack electrofishing unit was utilized by two people electrofishing from the lower to the upper boundaries of the sample segment. All fish species encountered were netted and placed in small net pens placed in the stream. We made two or three electrofishing passes, removing and segregating the fish from each pass. If no redband trout were encountered on the first pass and collection conditions were considered good, no further electrofishing passes were completed. All trout collected were measured to the nearest $\mathrm{mm}$ and weighed to the nearest gram. Trout were released after data collection. All other fish were identified to species, counted and released.

Redband trout population estimates and confidence intervals were calculated utilizing the MicroFish 3.0 program developed by Van Deventer and Platts (1987). Population estimates were calculated for all trout captured and for all trout greater than $100 \mathrm{~mm}$ in length, giving two estimates for sites where trout were collected. Trout densities were calculated by dividing the population estimate by the sample area and reported as trout $/ 100 \mathrm{~m}^{2}$.

\section{Stream Habitat}

Each stream segment was divided into ten equal length sections starting from the bottom. At each cross section, depth measurements were taken at $1 / 4,1 / 2$, and $3 / 4$ widths across the channel. Substrate composition was determined with standard IDFG methods, categorizing the substrate into size classes (Petrosky and Holubetz, 1988).

Instream fish cover was a subjective visual assessment of several parameters and was recorded for each cross-section as the percentage of the stream width defined as cover. For this study, cover was defined as areas where redband trout were likely to be found: (1) pools $>0.45 \mathrm{~m}$ (>1.5 feet) in depth, (2) overhanging bank vegetation, (3) instream vegetation, (4) near large instream rocks, (5) velocity breaks ie. broken water surface (6) pocket water behind or beside large rocks, (7) near large woody debris. 

Stream gradient was measured using an ocular hand level and a stadia rod. Gradient is the vertical drop between the upstream and downstream boundaries divided by the stream segment length and reported as a percentage.

Thermal input to the stream waters was measured using a Solar Pathfinder ${ }^{\mathrm{TM}}$ following methodologies outlined in Platts et. al. (1987). Percent stream shading was reported as the average percent of shading on the stream surface during June through September at 10 cross sections.

\section{Water Quality}

Several water quality parameters were measured at each stream segment. Conductivity and $\mathrm{pH}$ measurements were taken with hand held conductivity and $\mathrm{pH}$ meters. Hardness measurements were taken with Hach Company field titration kit. Water temperature was recorded with a pocket thermometer at each site.

Recording thermographs (HOBOS) were placed in 8 stream segments; 2 in Sinker Creek; 3 in the Reynolds watershed; 2 in the Squaw Creek watershed; and one at the Mud Flat Road crossing of Deep Creek. The HOBOS were placed in pools or runs to prevent dewatering and/or vandalism.

\section{RESULTS AND DISCUSSION}

\section{Redband Trout Populations}

\section{Trout Densities}

Ten of seventeen sampled stream segments contained redband trout in 1997 (Table 2). Seven of the sample sites had been sampled historically (BLM file data), and seven sites had been sampled since 1993 in conjunction with this project. Densities of redband trout ranged from 0 to $31.0 / 100 \mathrm{~m}^{2}$ for trout $>100 \mathrm{~mm}$ (Table 2). Generally an increase in density has occurred at sites since the drought ended.

\section{Jordan Creek Drainage}

Three Jordan Creek sites, and one site on Flint Creek were resampled in 1997. The 1997 densities were higher than any documented in the previous two samples (Table 2). At sites JORDA095.4 and FLINT003.9 redband trout densities have increased in both samples taken in the 1990's when compared to the historical 1977 data. The two other Jordan Creek sites had lower trout densities in 1993 from the historic sampling but 

rebounded to higher densities in 1997 (Table 2).

\section{Squaw Creek Drainage}

No redband trout were found at the three sites in 1997. There is no record of historical fish sampling in this drainage.

\section{Reynolds Creek Drainage}

Three of the four Reynolds Creek drainage stream segments sampled contained redband trout in 1997 (REYNO023.7,REYNO006.6, and SALMO000.6). Sample site MACKS002.0 did not have redbands (Table 2). There were major increases in the two Reynolds Creek sites from 1994 when there were zero redband captured at the sites (Table 2). The upper Reynolds Creek site REYNO023.7 was dry when visited in 1994. The Salmon Creek site may have helped the lower Reynolds Creek site repopulate since 1994.

\section{Sinker Creek Drainage}

Two sites were investigated in the Sinker drainage in 1997 (SINKE016.0 and SCBOB000.7). The Sinker Creek site was located approximately $0.6 \mathrm{~km}$ downstream of the road leading to Silver City. Redbands were collected at this site at a density of $2.5 / 100 \mathrm{M}^{2}$ for trout $>100 \mathrm{~mm}$. Sinker Creek was previously sampled downstream at stream mile 7.6 and was found to contain a density of redbands of $34.0 / 100 \mathrm{M}^{2}$. Scotch Bob Creek, a tributary to Sinker Creek, also contained a low density of redbands at $2.0 / 100 \mathrm{M}^{2}$ for trout $>100 \mathrm{~mm}$ (Table 2).

\section{Deep Creek Drainage}

Two sites were sampled in Deep Creek, a site just downstream of Mud Flat Road and a site at the confluence with the EF Owyhee River (DEEP_ 034.4 and DEEP 000.1). Neither of the sites contained redbands in 1997 at the time of sampling. The DEEP_034.4 site had been previously sampled in 1993 (this study) and in 1977. The 1977 survey documented 13.0 trout $/ 100 \mathrm{M}^{2}$ (Table 2). Sampling had been done at two other sites between these 1997 sites, and no redbands had been located ( Allen et al., 1994). One $300 \mathrm{~mm}$ redband was collected by angling in the Owyhee River just below the Deep Creek confluence during the July 1997 float trip.

\section{Red Canyon Drainage}

Sample site REDCA000.1 just above the confluence with the EF Owyhee River 

contained redband trout at a low density of $1.6 / 100 \mathrm{M}$ for trout $>100 \mathrm{~mm}$ (Table 2). Red Canyon Creek was sampled at several sites in 1994 and contained varying densities of redbands (Allen et al., 1994). Redband trout were observed in the EF Owyhee River near the mouth of Red Canyon Creek, during July, 1997.

\section{North Fork Owyhee River}

The NF Owyhee River was sampled downstream of the Mud Flat Road crossing and no redband trout were collected in 1997. A previous sampling in 1991 collected a few redband trout (Table 2).

\section{Owyhee River Float Trip}

Five biologists floated the Owyhee River in inflatable kayaks and rafts from the Garat put-in to the Three Forks take-out in eastern Oregon from July $7-15,1997$. We investigated all tributary confluences with the river. The Owyhee River was extremely low and at times several portages were necessary even with the small watercraft used on the trip. We extensively fished the river while paddling downstream, and only one redband trout was captured by angling in the Idaho reaches of the Owyhee River. Two tributaries were sampled via electrofishing methods, Deep Creek and Red Canyon Creek, previously described. The mouth of the South Fork Owyhee River was not sampled with electrofishing gear because it was deemed to deep to effectively sample, no fish were observed. The confluences of Piute Creek, Yatahoney Creek, Red Basin Creek, and Bald Mountain Creek were dry when visited. The angling catch was comprised of smallmouth bass and northern squawfish. Smallmouth bass were distributed along the entire reach floated. Our conclusion was that redband trout were almost entirely absent in these reaches of the Owyhee River.

\section{Redband Trout Length Frequencies}

Redband trout length frequencies for all sample sites are presented in Appendice 1. No age and growth information was collected in 1997.

\section{Nongame Fish Species Collected}

Nongame fish species were observed or captured at most sites. Species observed were: bridgelip sucker, Catostomus columbianus; Chislemouth, Acrocheilus alutaceus; longnose dace, Rhinichthys cataractae; Mottled sculpin, Cottus bairdi; redside shiner, 

Richardsonius balteatus; Smallmouth bass, Micropterus dolomieui; and speckled dace, Rhinichthys osculus. Species occurence and location is presented in Table 3.

\section{Habitat}

Habitat variables were collected consistent with previous survey years (Allen et al., 1994). As before, habitat data were collected to provide a baseline riparian habitat condition. Habitat variables of stream sample length, mean stream width, mean depth, percent gradient, and percent substrate composition are presented in Table 4. Table 5 contains the percent habitat type and percent trout cover observed. Percent stream shading derived from a Solar Pathfinder ${ }^{\mathrm{M}}$ are presented in Table 6.

\section{Water Quality}

The results of water quality measurements taken during 1997 are presented in Table 7. Water temperatures taken at time of fish sampling generally showed a higher trend than most previous samples. The stream sampling in 1997 was done mostly in the summer months while previous years data was collected later in the year, which may account for the higher water temperatures. Water temperatures although higher were still within acceptable limits for redband trout. Conductivity and $\mathrm{pH}$ were also elevated from previous survey ranges.

Eight recording thermographs (HOBOS) were placed in the study stream reaches near the sample sites in Squaw Creek (2), Salmon Creek, Macks Creek, Reynolds Creek, Sinker Creek (2), and Deep Creek. The thermograph data and locations and dates of deployment are provided in Appendice 2A - 2H. Macks Creek and Sinker Creek thermographs documented water temperatures above $25 \mathrm{C}$. Macks Creek did not contain redbands while the site on Sinker Creek near the thermograph did have a population of redband trout.

\section{CONCLUSION}

Ten of seventeen stream sites contained redband trout. Generally an increase in density has occurred since the drought of the late 1980's and early 1990's. In Deep Creek and the NF Owyhee River redbands were not located where previously found and may well be much reduced in abundance. The Owyhee River redband population seems much reduced from their previously estimated range (BLM data maps). The loss of redband trout populations fits with the data collected for this report, with their absence in the tributaries to the Owyhee River, and with their absence in the SF Owyhee River (Allen et 

al.,1997b) and Battle Creek (Allen et al., 1996). The pattern of redband trout population distribution is similar within the County, in that the redband populations are isolated from each other because the fish do not inhabit the larger streams and rivers anymore. This loss of connectivity between streams and their local redband populations is the greatest threat to the long-term persistence of these redband populations.

As suggested in Allen et al. (1996) a series of 5-20 ha riparian enclosures should be developed to allow the study of possible maximum densities of the these desert redband trout.

\section{RECOMMENDATIONS}

1. Establish several 5-20 hectare stream and riparian exclosures and monitor the changes to the riparian area, stream channel, and fish populations over time.

2. Publish a summary report of redband trout densities and estimated distributions found during these studies compared against the historical data.

3. Establish monitoring sites within each hydrologic unit within Owyhee County that can be resampled every five years for redband trout densities. 



\section{LITERATURE CITED}

Allen, D. B., B. J. Flatter, K. Fite, and S. P. Yundt. 1994. Redband trout (Oncorhynchusmykiss) population and habitat inventory in Owyhee County, Idaho. Idaho Department of Fish and Game, Bureau of Land anagement Challenge Cost Share Project, ID013-43500125-9Z.

Allen, D. B., B. J. Flatter, K. Fite. 1995. Redband trout (Oncorhynchus mykiss gairdneri) population and habitat surveys in Jump, Reynolds, and Sheep Creeks, and sections of the Owyhee River in Owyhee County, Idaho. Idaho Bureau of Land Management, Technical Bulletin No. 95-6.

Allen, D. B., K. Fite, J. Nelson, and B. J. Flatter. 1997a. Redband trout (Oncorhynchus mykiss gairdneri) population and habitat surveys in southern Owyhee County, Idaho. Idaho Bureau of Land Management, Technical Bulletin No. 97-10.

Allen, D. B., K. Fite, J. Nelson, B. J. Flatter. 1997b. Redband trout (Oncorhynchus mykiss gairdnerl) population and stream habitat surveys in Western Owyhee County, Idaho. Idaho Bureau of Land Management, Technical Bulletin No. 97-9.

Behnke, R.J. 1992. Native trout of western North America. American Fisheries Society Monograph 6.

Petrosky, C. E. and T. Holubetz. 1988. Idaho habitat evaluation for offsite mitigation record. Annual Report, 1987, Project 83-7 Dept. of Energy, BPA, Division of Fish and Wildlife.

Platts, W. S., C. Armour, G. D. Booth, M. Bryant, L. L. Bufford, P. Culpin, S. Jensen, G. W. Lienkaemper, G. W. Minshall, S. B. Monsen, R. L. Nelson, J. R. Sedell, and J. S. Tuhy. 1987. Methods for evaluating riparian habitats with applications to management. Gen. Tech. Report INT-221. U.S.D.A. Forest Service, Intermountain Research Station, Ogden, UT. $177 \mathrm{pp}$.

Van Deventer, J. S. and W. S. Platts. 1989. Microcomputer software system for generating population statistics from electrofishing data-users guide for MicroFish 3.O. U.S.D.A. Forest Service, Intermountain Research Station, Gen. Tech. Report INT-254. 

Table 1. Location and site descriptions of sites sampled in Owyhee County, Idaho in 1997.

\begin{tabular}{|c|c|c|c|}
\hline SITE & LOCATION & $\begin{array}{l}\text { LATITUDEI } \\
\text { LONGITUDE }\end{array}$ & SITE DESCRIPTION \\
\hline JORDA095.4 & T4S R3W S31 & $\begin{array}{l}\text { N } 431.98 \\
\text { W } 11644.75 \\
\end{array}$ & $\begin{array}{l}\text { Above first bridge } \\
\text { below Silver City }\end{array}$ \\
\hline JORDA088.3 & T4S R4W S31 & $\begin{array}{l}\text { N } 431.61 \\
\text { W } 11651 \\
\end{array}$ & $\begin{array}{l}\text { Just down stream from } \\
\text { Sage Hen Creek mouth }\end{array}$ \\
\hline JORDA075.9 & T6S R5W S1 & $\begin{array}{l}\text { N } 4256.04 \\
\text { W } 11652.69\end{array}$ & In canyon \\
\hline FLINT003.9 & T6S R4W S22 & $\begin{array}{l}\text { N } 4253.64 \\
\text { W } 11648.38 \\
\end{array}$ & $\begin{array}{l}\text { Above Triangle Road } \\
\text { crossing }\end{array}$ \\
\hline SQUAW008.7 & $\begin{array}{l}\text { T1N R5W S35 } \\
\text { NESE }\end{array}$ & $\begin{array}{l}\text { N } 4322.49 \\
\text { W } 11653.512 \\
\end{array}$ & $\begin{array}{l}1 / 4 \text { mile below private } \\
\text { fence }\end{array}$ \\
\hline SQUAW004.8 & $\begin{array}{l}\text { T1N R4W S8 } \\
\text { NWSE }\end{array}$ & $\begin{array}{l}\text { N } 4326.287 \\
\text { W } 11650.062 \\
\end{array}$ & $\begin{array}{l}\text { Above canyon near } \\
\text { only large cottonwood }\end{array}$ \\
\hline LSQUA000.2 & $\begin{array}{l}\text { T1N R5W S35 } \\
\text { NENE }\end{array}$ & $\begin{array}{l}\text { N } 4323.169 \\
\text { W } 11653.516\end{array}$ & $\begin{array}{l}100 \text { yds above } \\
\text { confluence into Squaw } \\
\mathrm{Cr} \text {. }\end{array}$ \\
\hline SALMO000.6 & T2S R4W S13 NWNE & $\begin{array}{l}\text { N } 4315.204 \\
\text { W } 11645.373 \\
\end{array}$ & $\begin{array}{l}1 / 4 \text { mile upstream of } \\
\text { gaging station }\end{array}$ \\
\hline MACKS002.0 & T2S R4W S27 NWNE & $\begin{array}{l}\text { N } 4313.62 \\
\text { W } 11647.758 \\
\end{array}$ & $50 \mathrm{~m}$ above diversion \\
\hline REYNO019.5 & T3S R4W S24 NESW & $\begin{array}{l}\text { N } 438.596 \\
\text { W } 11645.715 \\
\end{array}$ & $\begin{array}{l}150 \text { m below upper } \\
\text { gaging station }\end{array}$ \\
\hline REYNO011.3 & T2S R4W S12 SWNE & $\begin{array}{l}\text { N } 4315.847 \\
\text { W } 11645.102 \\
\end{array}$ & $\begin{array}{l}150 \mathrm{~m} \text { below lower } \\
\text { gaging station }\end{array}$ \\
\hline SCBOBO00.7 & T4S R3W S24 NESW & $\begin{array}{l}\text { N } 433.456 \\
\text { W } 11638.71 \\
\end{array}$ & $\begin{array}{l}0.7 \text { mi upstream of } \\
\text { Sinker Creek }\end{array}$ \\
\hline SINKE016.0 & T4S R2W S19 NENW & $\begin{array}{l}\text { N } 433.882 \\
\text { W } 11637.551 \\
\end{array}$ & $\begin{array}{l}1 / 2 \text { mi downstream of } \\
\text { upper road crossing }\end{array}$ \\
\hline DEEP_034.4 & T10S R3W S3 NWSE & $\begin{array}{l}\text { N } 4234.82 \\
\text { W } 11640.65\end{array}$ & $\begin{array}{l}200 \mathrm{~m} \text { below bridge on } \\
\text { Mud Flat road }\end{array}$ \\
\hline DEEP_000.1 & $\begin{array}{l}\text { T13S R3W S25 } \\
\text { NWSW }\end{array}$ & $N / A$ & $\begin{array}{l}\text { Mouth of Deep Creek } \\
\text { upstream }\end{array}$ \\
\hline REDCA000.1 & $\begin{array}{l}\text { T13S R4W S20 } \\
\text { NWNW }\end{array}$ & $\begin{array}{l}\text { N } 4216.96 \\
\text { W } 11650.36 \\
\end{array}$ & $\begin{array}{l}\text { From Owyhee River } \\
\text { upstream }\end{array}$ \\
\hline NFOWY011.8 & $\begin{array}{l}\text { T9S R5W S36 } \\
\text { SESE }\end{array}$ & $\begin{array}{l}\text { N } 4235.695 \\
\text { W } 11659.043\end{array}$ & $\begin{array}{l}150 \mathrm{~m} \text { below bridge at } \\
\text { the campground }\end{array}$ \\
\hline
\end{tabular}



Table 2. Redband trout population estimates and densities for stream sites sampled in Owyhee County, Idaho in 1997.

\begin{tabular}{|c|c|c|c|c|}
\hline SITE & $\begin{array}{l}\text { DATE } \\
\text { COLLECTED }\end{array}$ & $\begin{array}{l}\text { POPULATION } \\
\text { ESTIMATE (se) }\end{array}$ & $\begin{array}{l}\text { DENSITY } \\
\text { Number of } \\
{\text { Trout } 100 M^{2}}^{2}\end{array}$ & $\begin{array}{l}\text { DENSITY } \\
\text { Number of } \\
\text { Trout }>100 \mathrm{~mm} / \\
100 \mathrm{M}^{2}\end{array}$ \\
\hline JORDA095.4 & $9 / 4 / 97$ & $86(3.3)$ & 40.7 & 24.15 \\
\hline JORDA095.4 & $7 / 93$ & $54(4.9)$ & 15.5 & \\
\hline JORDA095.4 & $6 / 77$ & $86(16.2)$ & 10.2 & \\
\hline JORDA088.3 & $9 / 4 / 97$ & $73(2.2)$ & 20.9 & 14.4 \\
\hline JORDA088.3 & $8 / 93$ & $4(0.6)$ & 0.9 & \\
\hline JORDA088.3 & $8 / 76$ & $29(0)$ & 13.9 & \\
\hline JORDA075.9 & $9 / 5 / 97$ & $30(2.6)$ & 16.4 & 14.7 \\
\hline JORDA075.9 & $8 / 93$ & $4(0)$ & 1.2 & \\
\hline JORDA075.9 & $8 / 77$ & $6(0.9)$ & 3.2 & \\
\hline FLINT003.9 & $9 / 5 / 97$ & $94(8.7)$ & 104.1 & 31.0 \\
\hline FLINT003.9 & $9 / 93$ & $70(0.9)$ & 40.0 & \\
\hline FLINT003.9 & $7 / 77$ & $62(46.3)$ & 11.7 & \\
\hline SQUAW008.7 & $7 / 8 / 97$ & 0 & 0 & 0 \\
\hline SQUAW004.8 & $7 / 3 / 97$ & 0 & 0 & 0 \\
\hline LSQUA000.2 & $7 / 8 / 97$ & 0 & 0 & 0 \\
\hline SALMO000.6 & $7 / 9 / 97$ & $189(2.7)$ & 110.7 & 8.8 \\
\hline MACKS002.0 & 7/9/97 & 0 & 0 & 0 \\
\hline REYNO023.7 & $7 / 16 / 97$ & $44(1.9)$ & 20.0 & 20.0 \\
\hline REYNO023.7 & $9 / 27 / 94$ & O Dry & & \\
\hline REYNO023.7 & $7 / 13 / 77$ & $48(18.0)$ & 17.0 & \\
\hline REYNO006.6 & $7 / 16 / 97$ & $36(9.4)$ & 19.7 & 14.7 \\
\hline REYNO006.6 & $9 / 27 / 94$ & 0 & 0 & 0 \\
\hline
\end{tabular}





\begin{tabular}{|l|l|l|l|l|}
\hline SCBOB000.7 & $7 / 15 / 97$ & 2.0 & 2.0 & 2.0 \\
\hline SINKE016.0 & $7 / 15 / 97$ & $29(0.9)$ & 18.3 & 2.5 \\
\hline DEEP_034.4 & $8 / 25 / 97$ & 0 & 0 & 0 \\
\hline & $10 / 93$ & 0 & 0 & 0 \\
\hline DEEP_034.4 & & & 13.0 & \\
\hline DEEP_034.4 & $7 / 20 / 77$ & $19(4.4)$ & 0 & 0 \\
\hline DEEP_000.1 & $7 / 9 / 97$ & 0 & 5.7 & 1.6 \\
\hline REDCA000.1 & $7 / 11 / 97$ & $11(1.1)$ & 0 & 0 \\
\hline NFOWY011.8 & $7 / 10 / 97$ & 0 & 1.0 & \\
\hline NFOWY011.8 & $7 / 12 / 91$ & $2(0)$ & & \\
\hline
\end{tabular}




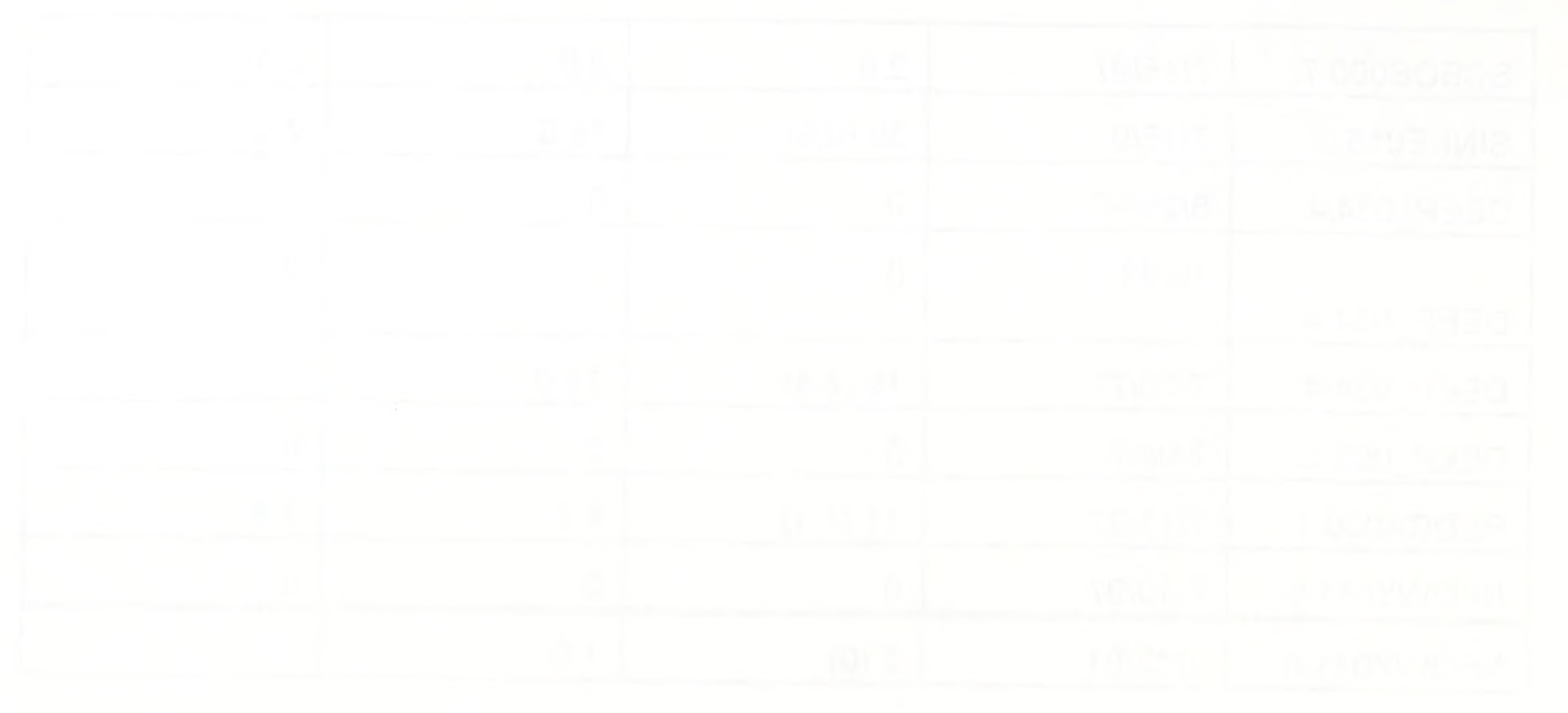


Table 3. Presence $(X)$ of fish species at sample sites in Owyhee County, Idaho in 1997.

\begin{tabular}{|c|c|c|c|c|c|c|c|c|c|c|}
\hline SITE & $\begin{array}{l}B \\
L \\
S\end{array}$ & $\begin{array}{l}\text { C } \\
S \\
L\end{array}$ & $\begin{array}{l}\text { L } \\
N \\
D\end{array}$ & $\begin{array}{l}M \\
T \\
S \\
\end{array}$ & $\begin{array}{l}R \\
S \\
S\end{array}$ & $\begin{array}{l}S \\
C \\
P\end{array}$ & $\begin{array}{l}\text { S } \\
\text { M } \\
\text { B }\end{array}$ & $\begin{array}{l}S \\
P \\
D\end{array}$ & $\begin{array}{l}S \\
U \\
K\end{array}$ & $\begin{array}{l}\text { W } \\
R \\
B\end{array}$ \\
\hline JORDA095.4 & & & & & & & & & & $x$ \\
\hline JORDA088.3 & $x$ & & & & & & & $x$ & & $x$ \\
\hline JORDA075.9 & $x$ & $x$ & $x$ & $x$ & $x$ & & & $x$ & & $x$ \\
\hline FLINT003.9 & & & & $x$ & & & & & & $x$ \\
\hline SQUAW008.7 & & & $x$ & & & & & & & \\
\hline SQUAW004.8 & $x$ & & $x$ & & & & & & & \\
\hline LSQUA000. 2 & & & & & & & & & & \\
\hline SALMO000.6 & & & & & & & & & & $x$ \\
\hline MACKSO02.0 & & & & & & & & & & \\
\hline REYNO019.5 & & & & & & & & $x$ & & $x$ \\
\hline REYNO011.3 & & & & & & & & $x$ & & $x$ \\
\hline SCBOBO00.7 & & & & & & & & & & $x$ \\
\hline SINKE016.0 & & & & & & & & & & $x$ \\
\hline DEEP_034.4 & $x$ & & & & $x$ & $x$ & & $x$ & & \\
\hline DEEP_000.1 & & $x$ & $x$ & & & $x$ & $x$ & & $x$ & \\
\hline REDCA000.1 & $x$ & & $x$ & & & $x$ & & & & $x$ \\
\hline NFOWY011.8 & & & $x$ & & & $x$ & & $x$ & & \\
\hline
\end{tabular}

BLS = Bridgelip sucker, $C S L=$ Chislemouth,$L N D=$ Longnose dace, MTS = Mottled sculpin, RSS = Redside shiner, SCP = Sculpin spp., SMB = Smalimouth bass, SPD = Speckled dace, SUK $=$ Sucker spp., WRB = Redband trout. 

Table 4. Stream sample site length, average width, average depth, percent gradient, and percent composition of substrate in stream sections sampled in Owyhee County, Idaho in 1997.

\begin{tabular}{|l|c|c|c|c|c|c|c|c|c|}
\hline SITE & $\begin{array}{c}\text { LE N } \\
(\mathrm{m})\end{array}$ & $\begin{array}{c}\text { A VE } \\
\text { WIDTH } \\
(\mathrm{m})\end{array}$ & $\begin{array}{c}\text { A VE } \\
\text { DEPTH } \\
(\mathrm{m})\end{array}$ & $\begin{array}{c}\% \\
\text { GRAD- } \\
\text { IENT }\end{array}$ & $\begin{array}{c}\% \\
\text { SAND }\end{array}$ & $\begin{array}{l}\% \\
\text { GRA- } \\
\text { VEL }\end{array}$ & $\begin{array}{l}\% \\
\text { RUBBLE }\end{array}$ & $\begin{array}{l}\% \\
\text { B OUL - } \\
\text { DER }\end{array}$ & $\begin{array}{l}\text { B } \\
\text { ROCK }\end{array}$ \\
\hline JORDA095.4 & 66 & 3.2 & 0.1 & $\mathrm{nd}$ & 26 & 12 & 54 & 7 & 0 \\
\hline JORDA088.3 & 66 & 5.3 & 0.1 & $\mathrm{nd}$ & 11 & 7 & 71 & 11 & 0 \\
\hline JORDA075.9 & 61 & 3.0 & 0.2 & $\mathrm{nd}$ & 15 & 28 & 49 & 9 & 0 \\
\hline FLINT003.9 & 43 & 2.1 & 0.2 & $\mathrm{nd}$ & 7 & 13 & 80 & 0 & 0 \\
\hline SQUAW008.7 & 61 & 1.9 & 0.1 & 1.02 & 21 & 13 & 47 & 19 & 0 \\
\hline SQUAW004.8 & 61 & 2.4 & 0.2 & 1.83 & 9 & 30 & 57 & 4 & 0 \\
\hline LSQUA000.2 & 61 & 1.0 & 0.1 & 2.11 & 17 & 12 & 72 & 0 & 0 \\
\hline SALMO000.6 & 61 & 2.8 & 0.1 & 1.76 & 14 & 3 & 74 & 10 & 0 \\
\hline MACKS002.0 & 61 & 2.0 & 0.1 & 1.31 & 11 & 24 & 42 & 23 & 0 \\
\hline REYNO019.5 & 61 & 3.6 & 0.2 & 1.81 & 0 & 19 & 62 & 16 & 3 \\
\hline REYNO011.3 & 61 & 3.0 & 0.2 & 0.73 & 12 & 30 & 43 & 16 & 0 \\
\hline SCBOB000.7 & 61 & 1.6 & 0.1 & 2.95 & 26 & 21 & 43 & 4 & 7 \\
\hline SINKE016.0 & 61 & 2.6 & 0.1 & 0.89 & 33 & 18 & 26 & 18 & 3 \\
\hline DEEP_034.4 & 61 & 3.9 & 0.1 & $\mathrm{nd}$ & 46 & 54 & 0 & 0 & 0 \\
\hline DEEP_000.1 & 300 & $\mathrm{nd}$ & $\mathrm{nd}$ & $\mathrm{nd}$ & $\mathrm{nd}$ & $\mathrm{nd}$ & $\mathrm{nd}$ & $\mathrm{nd}$ & $\mathrm{n}$ \\
\hline REDCA000.1 & 53 & $\mathrm{nd}$ & $\mathrm{nd}$ & $\mathrm{nd}$ & $\mathrm{nd}$ & $\mathrm{nd}$ & $\mathrm{nd}$ & $\mathrm{nd}$ & $\mathrm{n}$ \\
\hline NFOWY011.8 & 61 & 5.5 & 0.2 & 0.85 & 7 & 7 & 84 & 1 & 0 \\
\hline
\end{tabular}

nd $=$ no data 

Table 5. Percent habitat type and percent trout cover at stream sample sites in Owyhee County, Idaho in 1997.

\begin{tabular}{|c|c|c|c|c|c|}
\hline SITE & $\%$ POOL & $\%$ RIFFLE & $\%$ RUN & $\begin{array}{c}\% \text { POCKET } \\
\text { WATER }\end{array}$ & $\begin{array}{c}\% \text { TROUT } \\
\text { COVER }\end{array}$ \\
\hline JORDA095.4 & 0.0 & 53.3 & 46.7 & 0.0 & 10.0 \\
\hline JORDA088.3 & 26.7 & 53.3 & 20.0 & 0.0 & 17.0 \\
\hline JORDA075.9 & 40.0 & 60.0 & 0.0 & 0.0 & 32.0 \\
\hline FLINT003.9 & 40.0 & 26.7 & 33.3 & 0.0 & 0.0 \\
\hline SQUAW008.7 & 0.0 & 13.3 & 86.7 & 0.0 & 33.0 \\
\hline SQUAW004.8 & 0.0 & 50.0 & 50.0 & 0.0 & 50.0 \\
\hline LSQUA000.2 & 25.0 & 50.0 & 25.0 & 0.0 & 21.0 \\
\hline SALMO000.6 & 20.0 & 36.7 & 43.3 & 0.0 & 58.0 \\
\hline MACKS002.0 & 0.0 & 46.7 & 53.3 & 0.0 & 17.0 \\
\hline REYNO019.5 & 0.0 & 10.0 & 53.3 & 36.7 & 38.0 \\
\hline REYNO011.3 & 13.3 & 36.7 & 50.0 & 0.0 & 35.0 \\
\hline SCBOB000.7 & 10.0 & 30.0 & 20.0 & 40.0 & 20.0 \\
\hline SINKE016.0 & 0.0 & 20.0 & 50.0 & 30.0 & 7.0 \\
\hline DEEP_034.4 & 0.0 & 0.0 & 100.0 & 0.0 & 0.0 \\
\hline DEEP_000.1 & nd & nd & nd & nd & nd \\
\hline REDCA000.1 & nd & nd & nd & nd & nd \\
\hline NFOWY011.8 & 0.0 & 29.6 & 70.4 & 0.0 & 25.0 \\
\hline
\end{tabular}

nd $=$ no data 

Table 6. Percent stream shading on stream sites sampled in Owyhee County, Idaho in 1997.

\begin{tabular}{|l|l|}
\hline SITE & PERCENT SHADE \\
\hline JORDA095.4 & nd \\
\hline JORDA088.3 & nd \\
\hline JORDA075.9 & nd \\
\hline FLINT003.9 & nd \\
\hline SQUAW008.7 & 44.1 \\
\hline SQUAW004.8 & 53.8 \\
\hline LSQUA000.2 & 27.0 \\
\hline SALMO000.6 & 9.7 \\
\hline MACKS002.0 & 9.3 \\
\hline REYNO019.5 & nd \\
\hline REYNO011.3 & nd \\
\hline SCBOB000.7 & 53.7 \\
\hline SINKE016.0 & 18.7 \\
\hline DEEP_034.4 & 11.3 \\
\hline DEEP_000.1 & nd \\
\hline REDCA000.1 & nd \\
\hline NFOWY011.8 & 6.42 \\
\hline
\end{tabular}

$$
\text { nd }=\text { no data }
$$



Table 7. Water quality results for stream sites sampled in Owyhee County, Idaho in 1997.

\begin{tabular}{|c|c|c|c|c|c|}
\hline SITE & DATE & $\begin{array}{c}\text { WATER } \\
\text { TEMP } \\
\text { C }\end{array}$ & pH & $\begin{array}{c}\text { CONDUC- } \\
\text { TIVITY } \\
\text { Us/cm }\end{array}$ & $\begin{array}{c}\text { HARD- } \\
\text { NESS mg/l } \\
\text { as CaCO }\end{array}$ \\
\hline JORDA095.4 & $9 / 4 / 97$ & 17 & nd & nd & nd \\
\hline JORDA088.3 & $9 / 4 / 97$ & 20 & nd & nd & nd \\
\hline JORDA075.9 & $9 / 5 / 97$ & 12 & nd & nd & nd \\
\hline FLINT003.9 & $9 / 5 / 97$ & 16 & nd & nd & nd \\
\hline SQUAW008.7 & $7 / 8 / 97$ & 21 & 9.5 & 290 & 160 \\
\hline SQUAW004.8 & $7 / 3 / 97$ & 11 & 9.0 & 260 & 140 \\
\hline LSQUA000.2 & $7 / 8 / 97$ & 17.5 & 9.2 & 600 & 160 \\
\hline SALMO000.6 & $7 / 9 / 97$ & 19.5 & 9.6 & 330 & 187 \\
\hline MACKS002.0 & $7 / 9 / 97$ & 16 & 9.2 & 240 & 140 \\
\hline REYNO019.5 & $7 / 16 / 97$ & 17 & 9.3 & 90 & 85 \\
\hline REYNO011.3 & $7 / 16 / 97$ & 22 & 9.5 & 950 & 340 \\
\hline SCBOB000.7 & $7 / 15 / 97$ & 17 & 9.1 & 90 & 100 \\
\hline SINKE016.0 & $7 / 15 / 97$ & 18 & 8.9 & 100 & 102 \\
\hline DEEP_034.4 & $8 / 25 / 97$ & 20 & 9.3 & 50 & 60 \\
\hline DEEP_000.1 & $7 / 9 / 97$ & 18 & nd & nd & nd \\
\hline REDCA000.1 & $7 / 11 / 97$ & 17 & nd & nd & nd \\
\hline NFOWY011.8 & $7 / 10 / 97$ & 17 & 9.8 & 70 & 85 \\
\hline
\end{tabular}

nd $=$ no data 

APPENDICES 

Appendix 1. Stream site summaries for Owyhee County stream surveys done in 1997.

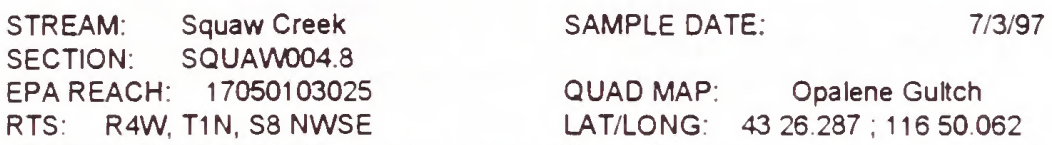

SECTION DESCRIPTION: Squaw Creek - Site begins above canyon near only large cottonwood.

Transect Information:

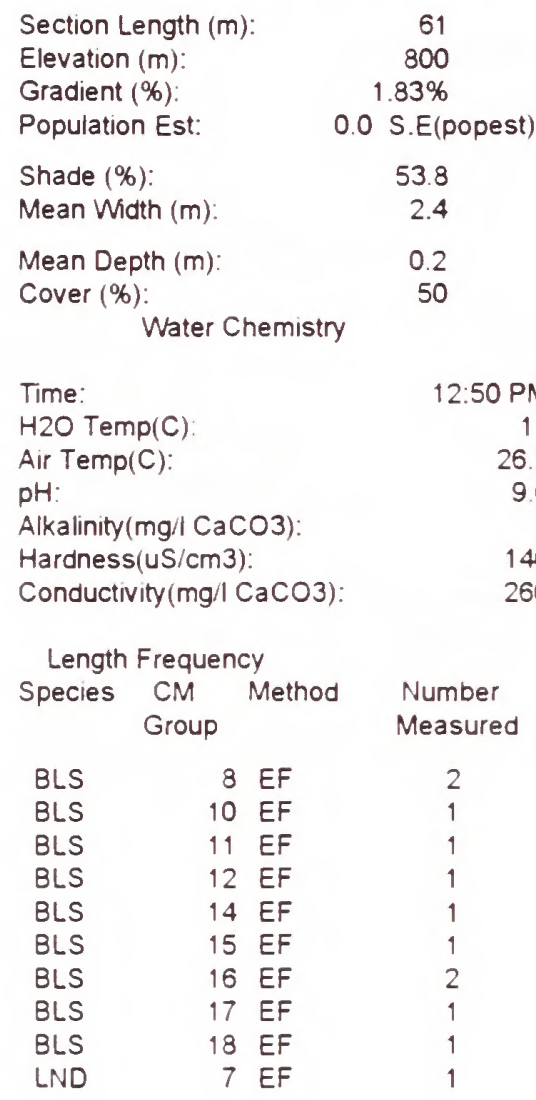

\begin{tabular}{|c|c|c|}
\hline \multicolumn{3}{|c|}{ Habitat Type: } \\
\hline Pool: & 0.0 & $\%$ \\
\hline Riffle: & 50.0 & $\%$ \\
\hline Run: & 50.0 & $\%$ \\
\hline Pocket: & 0.0 & $\%$ \\
\hline \multicolumn{3}{|c|}{ Substrate } \\
\hline Organic: & 0 & $\%$ \\
\hline Sand: & 9 & $\%$ \\
\hline Gravel: & 30 & $\%$ \\
\hline Rubble: & 57 & $\%$ \\
\hline Boulder: & 4 & $\%$ \\
\hline Bedrock: & 0 & $\%$ \\
\hline \multicolumn{3}{|l|}{ Species } \\
\hline BLS & Bridgelif & p sucker \\
\hline LND & Longno: & se dace \\
\hline
\end{tabular}



SECTION DESCRIPTION: Squaw Creek - $1 / 4$ mile below private fence.

Transect Information:

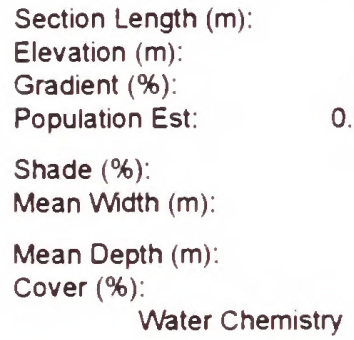

$\begin{array}{lrlr}\text { LND } & 5 & \text { EF } & 1 \\ \text { LND } & 6 & \text { EF } & 2 \\ \text { LND } & 7 & E F & 13 \\ \text { LND } & 8 & E F & 10 \\ \text { LND } & 9 & E F & 5 \\ \text { LND } & 10 \text { EF } & 2\end{array}$
10

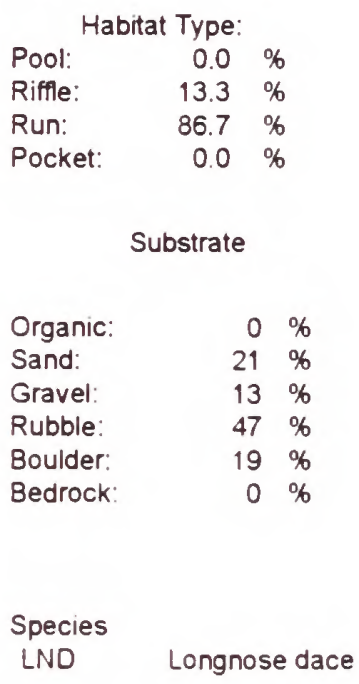



STREAM: Little Squaw Creek

SECTION: LSQUA000.2

EPA REACH: 17050103026

RTS: RSW, TIN, S35 NENE

SECTION DESCRIPTION: Little Squaw Creek - Section is 100 yds above confluence into Squaw Creek. The section ends at a natural barrier.
SAMPLE DATE:

$7 / 8 / 97$

\section{QUAD MAP: $\quad$ Jump Creek Canyon}

LAT/LONG: $\quad 4323.169 ; 11653.516$
Transect Information:

Section Length $(m)$ :

Elevation (m):

Gradient (\%):

Population Est:

Shade (\%):

Mean Width $(\mathrm{m})$ :

Mean Depth $(\mathrm{m})$ :

Cover (\%):

Water Chemistry

Time:

H2O Temp(C):

Air Temp(C):

$\mathrm{pH}$ :

Alkalinity(mg// $\mathrm{CaCO} 3$ ):

Hardness(uS/cm3)

Conductivity (mg// $\mathrm{CaCO} 3$ ):

Length Frequency

$\begin{array}{ccc}\text { Species } & \text { CM Method } & \text { Number } \\ \text { Group } & & \text { Measured }\end{array}$

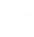

61
1125

$2.11 \%$

0.0 S.E(popest):

27.0

0.9

0.1

17

11:20 AM

17.5

31.5

9.2

160

600

ends at a natural barrier.

$\begin{array}{lrl} & \text { Habitat Type: } \\ \text { Pool: } & 20.0 & \% \\ \text { Riffle: } & 60.0 & \% \\ \text { Run: } & 20.0 & \% \\ \text { Pocket: } & 0.0 & \%\end{array}$

Substrate

\begin{tabular}{|c|c|}
\hline Organic: & 0 \\
\hline Sand: & 20 \\
\hline Gravel: & 19 \\
\hline Rubble: & 61 \\
\hline Boulder: & 0 \\
\hline Bedrock: & 0 \\
\hline
\end{tabular}

Species 

STREAM: Reynolds Creek

SECTION: REYNO011.3

EPA REACH: 17050103033

RTS: R4W, T2S, S12 SWNE

SECTION DESCRIPTION: Reynolds Creek - Section is located $\sim 150 \mathrm{~m}$ below the lower gauging station. Follow animal
SAMPLE DATE:

$7 / 16 / 97$

QUAD MAP: $\quad$ Rooster Comb Peak

LAT/LONG: $4315.847 ; 11645.102$
Transect Information:

Section Length (m):
Elevation $(\mathrm{m})$ :
Gradient (\%):
Population Est:
Shade (\%):
Mean Width (m):

Mean Depth (m):

Cover (\%):

Water Chemistry

Time:

H2O Temp(C):

Air Temp(C):

$\mathrm{pH}$ :

Alkalinity (mg// CaCO3):

Hardness(uS/cm3):

Conductivity(mg// $\mathrm{CaCO} 3$ ):

Length Frequency Species CM Method
Group

SPD

SPD

SPD

$S P D$

SPD

SPD

SPD

WRB

WRB

WRB

WRB

WRB

WRB

WRB

WRB

WRB

WRB

WRB

WRB

WRB

WRB

3 EF

$4 E F$

5 EF

6 EF

$7 \mathrm{EF}$

$8 \mathrm{EF}$

$9 \mathrm{EF}$

5 EF

$6 \mathrm{EF}$

7 EF

$8 \mathrm{EF}$

12 EF

13 EF

$15 \mathrm{EF}$

$16 \mathrm{EF}$

17 EF

18 EF

19 EF

20 EF

21 EF

25 EF

trail until it comes out a flat. The section begins there.

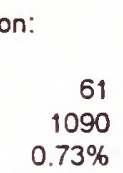

36.0 S.E(popest):

9

3.0

0.2

35

03:30 PM

22

31

9.5

340

950

Number

Measured

1
3
37
73
24
25
8
2
3
1
1
2
2
5
2
1
4
2
1
1
1

$\begin{array}{lrl} & \text { Habitat Type: } \\ \text { Pool: } & 13.3 & \% \\ \text { Riffle: } & 36.7 & \% \\ \text { Run: } & 50.0 & \% \\ \text { Pocket: } & 0.0 & \%\end{array}$

Substrate

$\begin{array}{lrl}\text { Organic: } & 0 & \% \\ \text { Sand: } & 12 & \% \\ \text { Gravel: } & 30 & \% \\ \text { Rubble: } & 43 & \% \\ \text { Boulder: } & 16 & \% \\ \text { Bedrock: } & 0 & \% \\ & \\ & \\ \text { Species } & \\ \text { SPD } & & \\ \text { WRB } & \text { Speckled dace } \\ & & \end{array}$



SECTION DESCRIPTION: Salmon Creek - $1 / 4$ mile upstream of gauging station.

Transect Information:

$\begin{array}{lr}\text { Section Length }(\mathrm{m}): & 6 \\ \text { Elevation }(\mathrm{m}): & 1150 \\ \text { Gradient }(\%): & 1.76 \% \\ \text { Population Est: } & 189.0 \mathrm{S.E} \\ \text { Shade (\%): } & 9.7 \\ \text { Mean Width }(\mathrm{m}): & 2.8 \\ \text { Mean Depth }(\mathrm{m}): & 0 . \\ \text { Cover }(\%): & 58\end{array}$

Water Chemistry

Time:

H2O Temp(C)

Air Temp(C):

pH:

Alkalinity (mg/l $\mathrm{CaCO})$

Hardness(uS/cm3)

Conductivity (mg// $\mathrm{CaCO} 3$ )

Length Frequency

\begin{tabular}{|c|c|c|}
\hline ies & $\begin{array}{l}\mathrm{CM} \\
\text { Group }\end{array}$ & Method \\
\hline
\end{tabular}

$\begin{array}{lrlr}\text { WRB } & 4 \text { EF } & 14 \\ \text { WRB } & 5 \text { EF } & 82 \\ \text { WRB } & 6 \text { EF } & 71 \\ \text { WRB } & 7 \text { EF } & 8 \\ \text { WRB } & 13 \text { EF } & 2 \\ \text { WRB } & 14 \text { EF } & 4 \\ \text { WRB } & 15 \text { EF } & 1 \\ \text { WRB } & 16 \text { EF } & 2 \\ \text { WRB } & 17 \text { EF } & 1 \\ \text { WRB } & 18 \text { EF } & 3 \\ \text { WRB } & 20 \text { EF } & 2\end{array}$

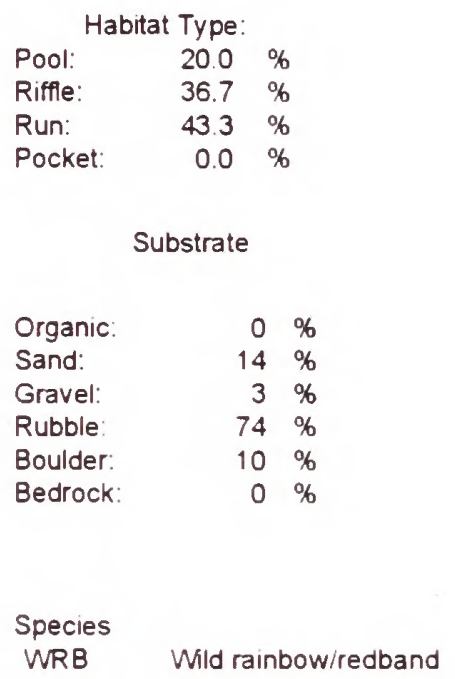

WRB

Wild rainbow/redband 

STREAM: Macks Creek

SECTION: MACKSOO2.0

EPA REACH: $\quad 17050103036$

RTS: R4W, T2S, S27 NWNE

LAT/LONG: $4313.62 ; 11647.758$

\section{QUAD MAP: Roostercomb Peak}

Macks Creek - Section is located $\sim 50 \mathrm{~m}$ above the diversion. There is an old jeep trail that can be taken all the way to it.
Transect Information:

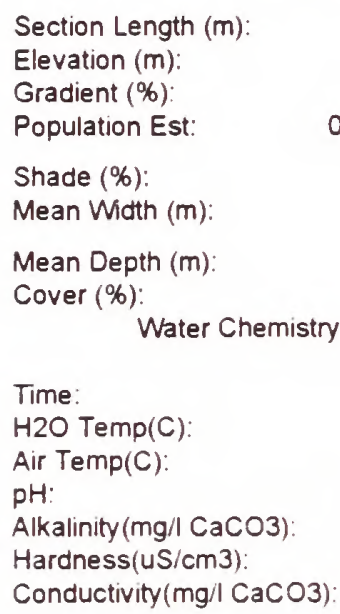

$\begin{array}{lrc} & \text { Habitat Type: } \\ \text { Pool: } & 0.0 & \% \\ \text { Riffle: } & 46.7 & \% \\ \text { Run: } & 53.3 & \% \\ \text { Pocket: } & 0.0 & \%\end{array}$

\section{Substrate}

\begin{tabular}{|c|c|}
\hline Organic: & 0 \\
\hline Sand: & 11 \\
\hline Gravel: & 24 \\
\hline Rubble: & 42 \\
\hline Boulder: & 23 \\
\hline Bedrock: & 0 \\
\hline
\end{tabular}

Species

$$
\begin{array}{r}
16 \\
22 \\
9.2 \\
140 \\
240
\end{array}
$$

Measured 

STREAM: Reynolds Creek

SECTION: REYNO019.5

EPA REACH: 17050103037

RTS: R4W, T3S, S24 NESW
$7 / 16 / 97$

SAMPLE DATE:

QUAD MAP: $\quad$ Soilder Cap

LAT/LONG: $\quad 438.596 ; 11645.715$

Reynolds Creek - Section is located $\sim 150 \mathrm{~m}$ below the upper gauging station at the first bend where the water channel narrows, then widens again.

Transect Information

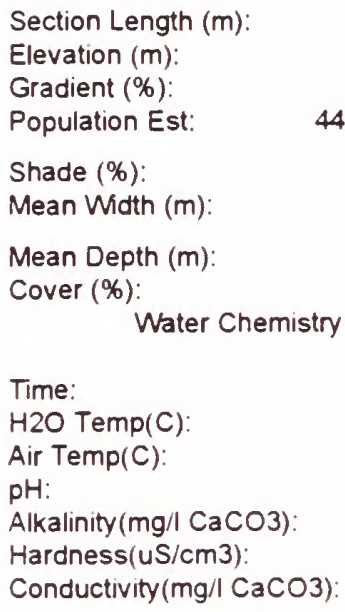

$\begin{array}{lrl} & \text { Habitat Type: } \\ \text { Pool: } & 0.0 & \% \\ \text { Riffle: } & 10.0 & \% \\ \text { Run: } & 53.3 & \% \\ \text { Pocket: } & 36.7 & \%\end{array}$

\section{Substrate}

\begin{tabular}{|c|c|c|}
\hline Organic: & 0 & $\%$ \\
\hline Sand: & 0 & $\%$ \\
\hline Gravel: & 19 & $\%$ \\
\hline Rubble: & 62 & $\%$ \\
\hline Boulder: & 16 & $\%$ \\
\hline Bedrock: & 3 & $\%$ \\
\hline \multicolumn{3}{|l|}{ Species } \\
\hline $\begin{array}{l}\text { SPD } \\
\text { WRB }\end{array}$ & $\begin{array}{l}\text { Speckle } \\
\text { Wild rain }\end{array}$ & $\begin{array}{l}\text { d dace } \\
\text { bow/redband }\end{array}$ \\
\hline
\end{tabular}

Number Measured
12:00 PM

\section{2}





\begin{tabular}{|c|c|c|c|}
\hline STREAM: & Sinker Creek & SAMPLE DATE & $7 / 15 / 97$ \\
\hline SECTION: & SINKE016.0 & & \\
\hline EPA REACH: & 17050103048 & QUAD MAP: & Sinker Canyon \\
\hline R2W. & T4S, S19 NENW & LAT/LONG: 4 & $433.882 ; 11637.551$ \\
\hline
\end{tabular}

Transect Information:

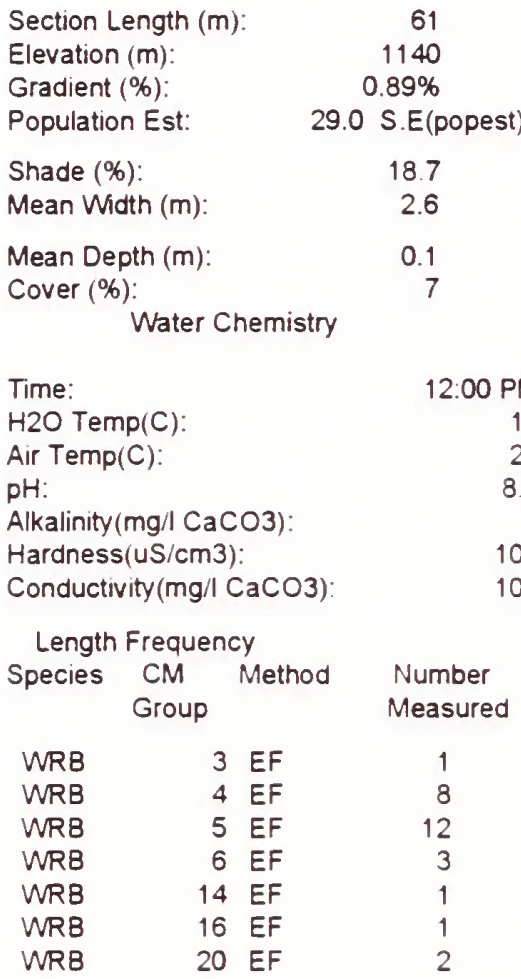

\begin{tabular}{|c|c|c|}
\hline \multicolumn{3}{|c|}{ Habitat Type: } \\
\hline Pool: & 0.0 & \\
\hline Riffle: & 20.0 & \\
\hline Run: & 50.0 & \\
\hline Pocket: & 30.0 & \\
\hline
\end{tabular}

Substrate

$\begin{array}{lrl}\text { Organic: } & 0 & \% \\ \text { Sand: } & 33 \% \\ \text { Gravel: } & 18 \% \\ \text { Rubble: } & 26 \% \\ \text { Boulder: } & 18 \% \\ \text { Bedrock: } & 3 \% \\ & \\ & \\ \text { Species } & \\ \text { WRB } & \text { Wild rainbow/redband }\end{array}$



STREAM: Scotch Bob Creek

SECTION: SCBOBO00.7

EPA REACH: 17050103090

RTS: R3W, T4S, S24 NESW

SECTION DESCRIPTION: Scotch BOb Cree
$7 / 15 / 97$

QUAD MAP: $\quad$ Sitver City

LAT/LONG: $\quad 433.456 ; 11638.71$

- 7 mile upstream of Sinker $\mathrm{Cr}$.
Transect Information:

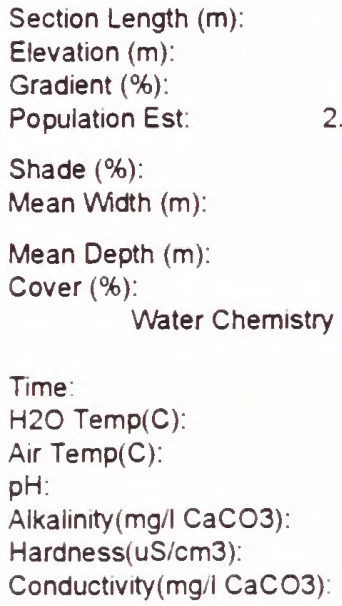

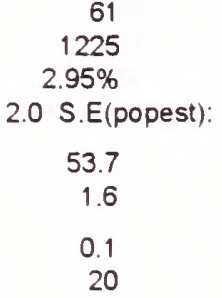



SECTION DESCRIPTION: Deep Creek - Mouth of Deep Cr. upstream.

Transect Information:

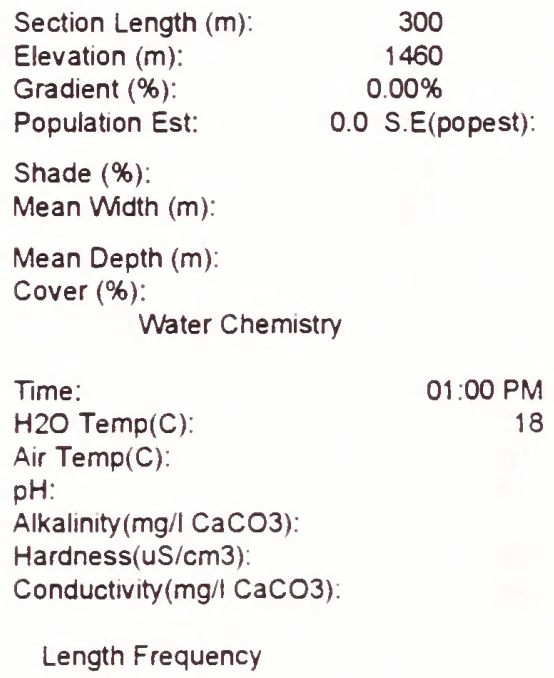

300

1460

$0.00 \%$

0.0 S.E(popest):

\section{$01: 00 \mathrm{PM}$}

18

0

SUK Sucker spp

$\begin{array}{lrll}\text { CSL } & 7 & \text { EF } & 1 \\ \text { CSL } & 9 & \text { EF } & 1 \\ \text { LND } & 8 & \text { EF } & 2 \\ \text { SCP } & 3 \text { EF } & 1 \\ \text { SCP } & 6 \text { EF } & 1 \\ \text { SCP } & 7 \text { EF } & 2 \\ \text { SMB } & 7 \text { EF } & 1 \\ \text { SMB } & 9 \text { EF } & 2 \\ \text { SMB } & 11 \text { EF } & 2 \\ \text { SMB } & 12 \text { EF } & 1 \\ \text { SMB } & 15 \text { EF } & 1 \\ \text { SUK } & 9 \text { EF } & 1 \\ \text { SUK } & 14 \text { EF } & 1\end{array}$



STREAM: Deep Creek

SECTION: DEEP_034.4

EPA REACH: $\quad 17050104101$

RTS: R3W, T1OS, S3 NWSE

R3W, T1OS, S3NWSE

the section is a barbwire fence that marks the private ground

Transect Information:

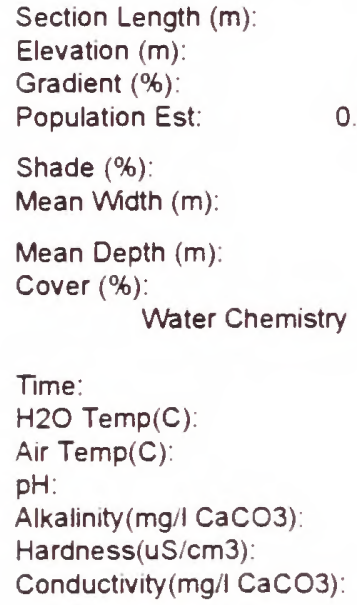

$\begin{array}{lrl} & \text { Habitat Type: } \\ \text { Pool: } & 0.0 & \% \\ \text { Riffle: } & 0.0 & \% \\ \text { Run: } & 100.0 & \% \\ \text { Pocket: } & 0.0 & \%\end{array}$

\section{Substrate}

$$
3.9
$$

0

12:30 PM

20

Organic:

Sand:

Gravel:

Rubble:

Boulder:

Bedrock:

$\begin{array}{rr}0 & \% \\ 46 & \% \\ 54 & \% \\ 0 & \% \\ 0 & \% \\ 0 & \%\end{array}$

Species

BLS Bridgelip sucker

RSS Redside shiner

SCP Sculpin spp.

SPD Speckled dace 

STREAM: Red Canyon Creek

SECTION: REDCAOOO.1

EPA REACH: 17050104111

RTS: R4W, T13S, S20 NWNW

SECTION DESCRIPTION: Red Canyon Creek - From Owyhee River upstream.
$7 / 11 / 97$

$\begin{array}{lr}\text { QUAD MAP: } & \text { Red Basin Idaho } \\ \text { LAT/LONG: } 4216.96 ; 11650.36\end{array}$

LAT/LONG: $4216.96 ; 11650.36$
Transect Information:

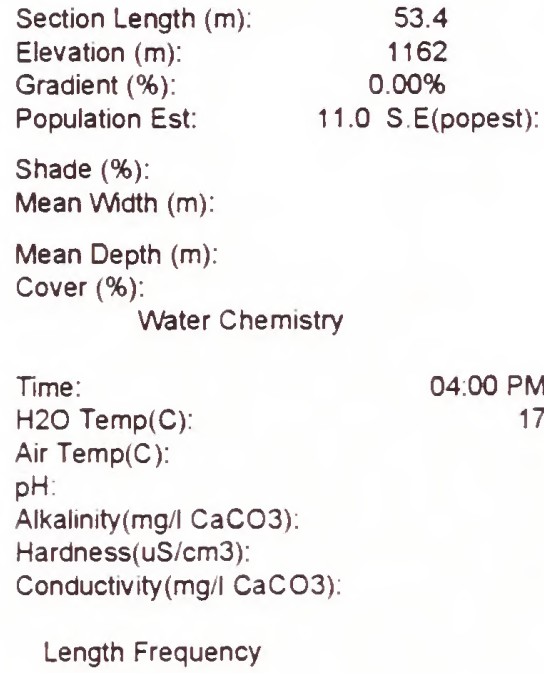

Habitat Type:

$\begin{array}{ll}\text { Pool: } & \% \\ \text { Riffle: } & \% \\ \text { Run: } & \% \\ \text { Pocket: } & \%\end{array}$

\section{Substrate}

$\begin{array}{ll}\text { Organic: } & \% \\ \text { Sand: } & \% \\ \text { Gravel: } & \% \\ \text { Rubble: } & \% \\ \text { Boulder: } & \% \\ \text { Bedrock: } & \%\end{array}$

$\begin{array}{ll}\begin{array}{ll}\text { Species } \\ \text { BLS }\end{array} & \text { Bridgelip sucker } \\ \text { LND } & \text { Longnose dace } \\ \text { SCP } & \text { Sculpin spp. } \\ \text { WRB } & \text { Wild rainbow/redband }\end{array}$



STREAM: Owyhee River, NF

SECTION: NFOWY011.8

EPA REACH: 17050107043

RTS: R5W, T9S, S36 SESE

LAT/LONG: $4235.695 ; 11659.043$

$7 / 10 / 97$

Fairylawn

N.F. Owyhee River - Section is located $\sim 150 \mathrm{~m}$ below bridge at the campround. The site runs alongside a large gravelbar.

Transect Information:

\section{Section Length (m) \\ Elevation $(\mathrm{m})$ : \\ Gradient (\%): \\ Population Est:}

Shade (\%):

Mean Width (m):

Mean Depth $(\mathrm{m})$ :

Cover (\%):

Water Chemistry

Time:

H2O Temp(C):

Air Temp(C):

pH:

Alkalinity (mg/l $\mathrm{CaCO}$ )

Hardness(uS/cm3):

Conductivity (mg// $\mathrm{CaCO} 3$ )

Length Frequency

$\begin{array}{ccc}\text { Species } & \mathrm{CM} \\ \text { Group } & \text { Method } & \begin{array}{l}\text { Number } \\ \text { Measured }\end{array}\end{array}$

\begin{tabular}{|c|c|c|}
\hline LND & 4 & $E F$ \\
\hline LND & 5 & $E F$ \\
\hline LND & 6 & $E F$ \\
\hline LND & 7 & $E F$ \\
\hline LND & 8 & $E F$ \\
\hline LND & 9 & $E F$ \\
\hline SCP & 5 & $E F$ \\
\hline $\mathrm{SCP}$ & 6 & $E F$ \\
\hline$S C P$ & 7 & $E F$ \\
\hline SPD & 5 & $E F$ \\
\hline SPD & 6 & $E F$ \\
\hline SPD & 7 & $E F$ \\
\hline SPD & 10 & $E F$ \\
\hline
\end{tabular}

$\begin{array}{lrc} & \text { Habitat Type: } \\ \text { Pool: } & 0.0 & \% \\ \text { Riffle: } & 26.7 & \% \\ \text { Run: } & 73.3 & \% \\ \text { Pocket: } & 0.0 & \%\end{array}$

Substrate

\begin{tabular}{|c|c|c|}
\hline Organic: & 0 & $\%$ \\
\hline Sand: & 7 & $\%$ \\
\hline Gravel: & 6 & $\%$ \\
\hline Rubble: & 86 & $\%$ \\
\hline Boulder: & 1 & $\%$ \\
\hline Bedrock: & 0 & $\%$ \\
\hline \multicolumn{3}{|l|}{ Species } \\
\hline LND & \multicolumn{2}{|c|}{ Longnose dace } \\
\hline SCP & \multicolumn{2}{|c|}{ Sculpin spp. } \\
\hline SPD & \multicolumn{2}{|c|}{ Speckled dace } \\
\hline
\end{tabular}



STREAM: Jordan Creek

SECTION: JORDA075.9

EPAREACH: $\quad 17050108055$

RTS: R, T, S

SECTION DESCRIPTION: In canyon
SAMPLE DATE:

$9 / 5 / 97$

QUAD MAP: Triangle

LAT/LONG: $\quad 4256.04 ; 11652.69$

Transect Information:

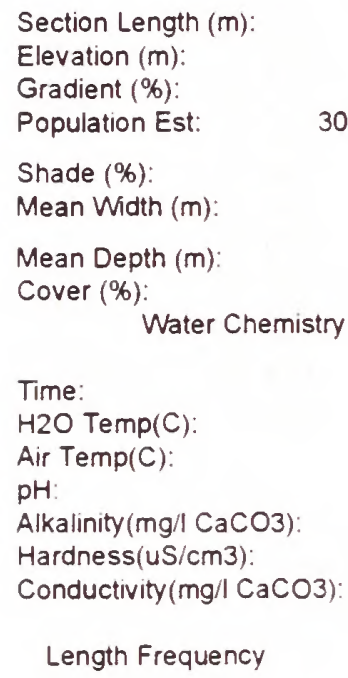

$\begin{array}{lrll}\text { BLS } & 3 & \text { ef-3 } & 3 \\ \text { BLS } & 4 & \text { ef-3 } & 1 \\ \text { BLS } & 6 \text { ef-3 } & 2 \\ \text { BLS } & 7 \text { ef-3 } & 1 \\ \text { BLS } & 8 \text { ef-3 } & 3 \\ \text { BLS } & 9 \text { ef-3 } & 6 \\ \text { BLS } & 10 \text { ef-3 } & 2 \\ \text { BLS } & 11 \text { ef-3 } & 5 \\ \text { BLS } & 12 \text { ef-3 } & 5 \\ \text { BLS } & 13 \text { ef-3 } & 6 \\ \text { BLS } & 15 \text { ef-3 } & 2 \\ \text { CSL } & 6 \text { ef-3 } & 1 \\ \text { CSL } & 10 \text { ef-3 } & 3 \\ \text { CSL } & 11 \text { ef-3 } & 1 \\ \text { CSL } & 16 \text { ef-3 } & 1 \\ \text { LND } & 5 \text { ef-3 } & 1 \\ \text { LND } & 6 \text { ef-3 } & 7 \\ \text { LND } & 7 & \text { ef-3 } & 3 \\ \text { LND } & 8 \text { ef-3 } & 1 \\ \text { LND } & 9 \text { ef-3 } & 1\end{array}$

$\begin{array}{lrl} & \text { Habitat Type: } \\ \text { Pool: } & 40.0 & \% \\ \text { Riffle: } & 60.0 & \% \\ \text { Run: } & 0.0 & \% \\ \text { Pocket: } & 0.0 & \%\end{array}$

Substrate

$\begin{array}{lr}\text { Organic: } & 0 \% \\ \text { Sand: } & 15 \% \\ \text { Gravel: } & 28 \% \\ \text { Rubble: } & 49 \% \\ \text { Boulder: } & 9 \% \\ \text { Bedrock: } & 0 \% \\ & \\ & \\ \text { Species } & \\ \text { BLS } & \text { Bridgelip sucker } \\ \text { CSL } & \text { Chiselmouth } \\ & \\ \text { LND } & \text { Longnose dace } \\ \text { MTS } & \text { Mottled sculpin } \\ \text { RSS } & \text { Redside shiner } \\ \text { SPD } & \text { Speckled dace } \\ \text { WRB } & \text { Wild rainbow/redband }\end{array}$





\begin{tabular}{|c|c|c|c|}
\hline MTS & 4 & ef-3 & 2 \\
\hline MTS & 6 & ef-3 & 1 \\
\hline MTS & 7 & ef-3 & 1 \\
\hline RSS & 7 & ef-3 & 1 \\
\hline RSS & 8 & ef-3 & 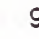 \\
\hline RSS & 9 & ef-3 & 6 \\
\hline RSS & 10 & ef-3 & 3 \\
\hline SPD & 2 & ef-3 & 1 \\
\hline SPD & 3 & ef-3 & 1 \\
\hline SPD & 4 & ef-3 & 3 \\
\hline SPD & 5 & ef-3 & 13 \\
\hline SPD & 6 & ef-3 & 33 \\
\hline SPD & 7 & ef-3 & 10 \\
\hline SPD & 8 & ef-3 & 1 \\
\hline WRB & 5 & ef-3 & 2 \\
\hline WRB & 10 & ef-3 & 3 \\
\hline WRB & 11 & ef-3 & 4 \\
\hline WRB & 12 & ef-3 & 5 \\
\hline WRB & 13 & ef-3 & 5 \\
\hline WRB & 14 & ef-3 & 1 \\
\hline WRB & 17 & ef-3 & 1 \\
\hline WRB & 19 & ef-3 & 3 \\
\hline WRB & 20 & ef-3 & 2 \\
\hline WRB & 25 & ef-3 & 2 \\
\hline
\end{tabular}



SECTION DESCRIPTION: Just down stream from Sage Hen Creek mouth.

Transect Information:

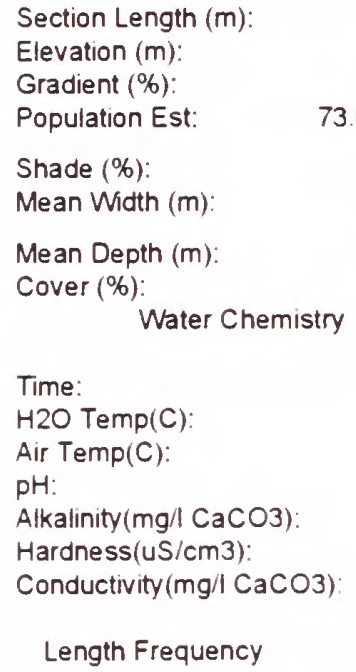

$\begin{array}{lrr} & \text { Habitat Type: } \\ \text { Pool: } & 26.7 & \% \\ \text { Riffle: } & 53.3 & \% \\ \text { Run: } & 20.0 & \% \\ \text { Pocket: } & 0.0 & \%\end{array}$

Substrate

\begin{tabular}{|c|c|}
\hline Organic: & 0 \\
\hline Sand: & 11 \\
\hline Gravel: & 7 \\
\hline Rubble: & 71 \\
\hline Boulder: & 11 \\
\hline Bedrock: & 0 \\
\hline
\end{tabular}

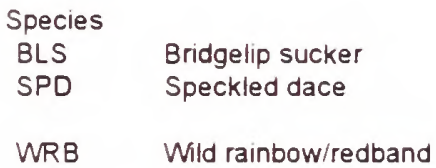



STREAM: Jordan Creek

SECTION DESCRIPTION: Above first bridge below Silver City

Transect Information:

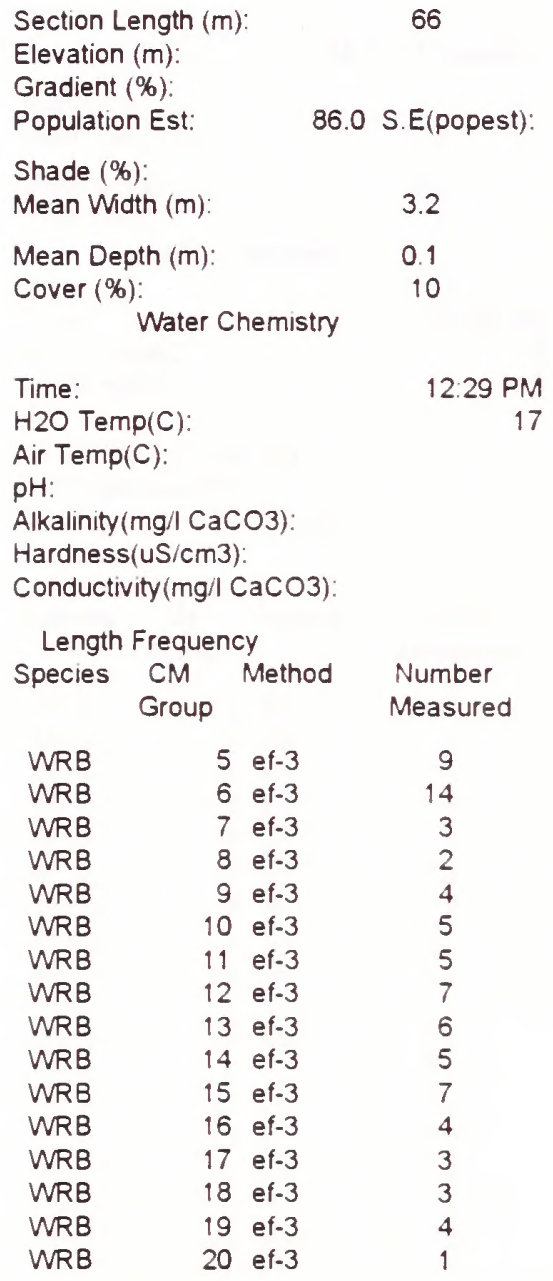

$\begin{array}{lrl} & \text { Habitat Type: } \\ \text { ol: } & 0.0 & \% \\ \text { n: } & 53.3 & \% \\ \text { cket: } & 46.7 & \% \\ & 0.0 & \%\end{array}$

\section{Substrate}

$\begin{array}{lrl}\text { Organic: } & 0 & \% \\ \text { Sand: } & 26 & \% \\ \text { Gravel: } & 12 & \% \\ \text { Rubble: } & 54 & \% \\ \text { Boulder: } & 7 & \% \\ \text { Bedrock: } & 1 & \% \\ & & \\ & & \\ \text { Species } & & \\ \text { WRB } & \text { Wild rainbow/redband }\end{array}$



SECTION: FLINTO03.9

EPA REACH: 17050108079

QUAD MAP: Triangle

RTS: R4W, T6S, S22

LAT/LONG: $4253.64 ; 11648.38$

SECTION DESCRIPTION: Above Triangle Road Crossing

Transect Information:

Section Length $(m)$ :

43

Elevation $(m)$ :

Gradient (\%):

Population Est:

94.0 S.E(popest):

9

Shade (\%):

Mean Width $(\mathrm{m})$ :

Mean Depth (m)

Cover (\%):

Water Chemistry

Time:

H2O Temp $(C)$ :

Air Temp(C):

$\mathrm{pH}$ :

Alkalinity (mg/l $\mathrm{CaCO} 3)$

Hardness(uS/cm3):

Conductivity (mg// $\mathrm{CaCO} 3$ )

Length Frequency Species CM Method
Group

$01: 00 \mathrm{PM}$
16

$01: 00 \mathrm{PM}$
16

0.2

0
2 ef-

5 ef-

6 ef-

7 ef-

8 ef-

9 ef-

4 ef-

5 ef-

6 ef-

7 ef-

8 ef-

9 ef-

10 ef-

11 ef-

13 ef-

14 ef-

15 ef-

16 ef-

17 ef-

18 ef-

20 ef-

23 ef-

Number

Measured

21

16

4

3

3

3

7

4

6

6
2

WRB

WRB

WRB

WRB
1

1

1

6

1
2
1
4
2
1
6
1
6
4
3
3
3
7
4
6
2
1
1
1
1
1

$\begin{array}{lrl} & \text { Habitat Type: } \\ \text { Pool: } & 40.0 & \% \\ \text { Riffle: } & 26.7 & \% \\ \text { Run: } & 33.3 & \% \\ \text { Pocket: } & 0.0 & \%\end{array}$

\section{Substrate}

\begin{tabular}{|c|c|}
\hline Organic: & 0 \\
\hline Sand: & 7 \\
\hline Gravel: & 13 \\
\hline Rubble: & 80 \\
\hline Boulder: & 0 \\
\hline Bedrock: & 0 \\
\hline
\end{tabular}

Species

MTS Mottled sculpin

WRB Wild rainbow/redband 



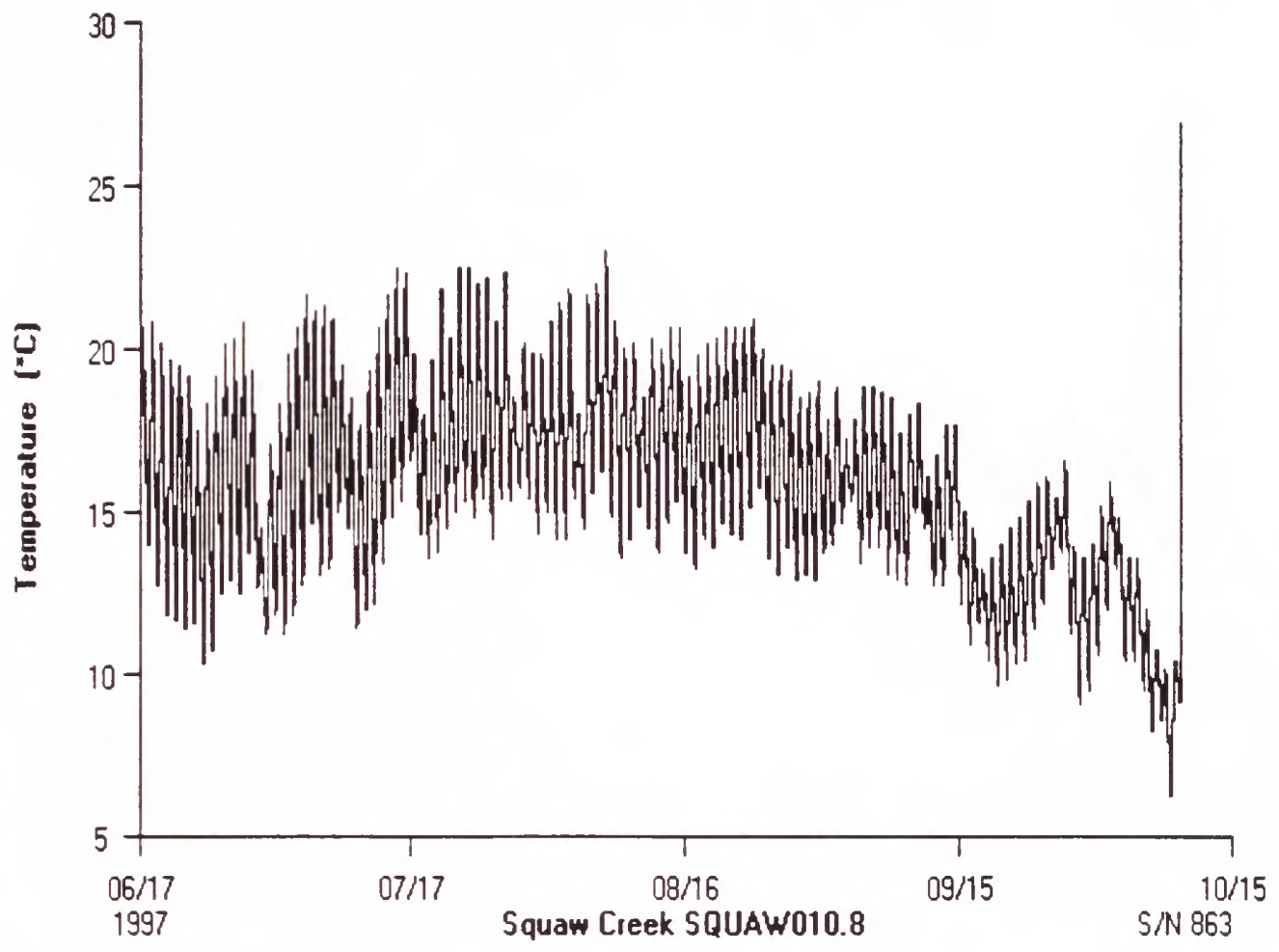

Appendix 2A.

Recording thermograph data for Squaw Creek, Owyhee County, ID. Site is located at Stream Mile 10.8, Legal Description T1N R5W S36 SWSW, Elevation 1175m. Thermograph was set on 6/17/97 and pulled on 10/15/97. 



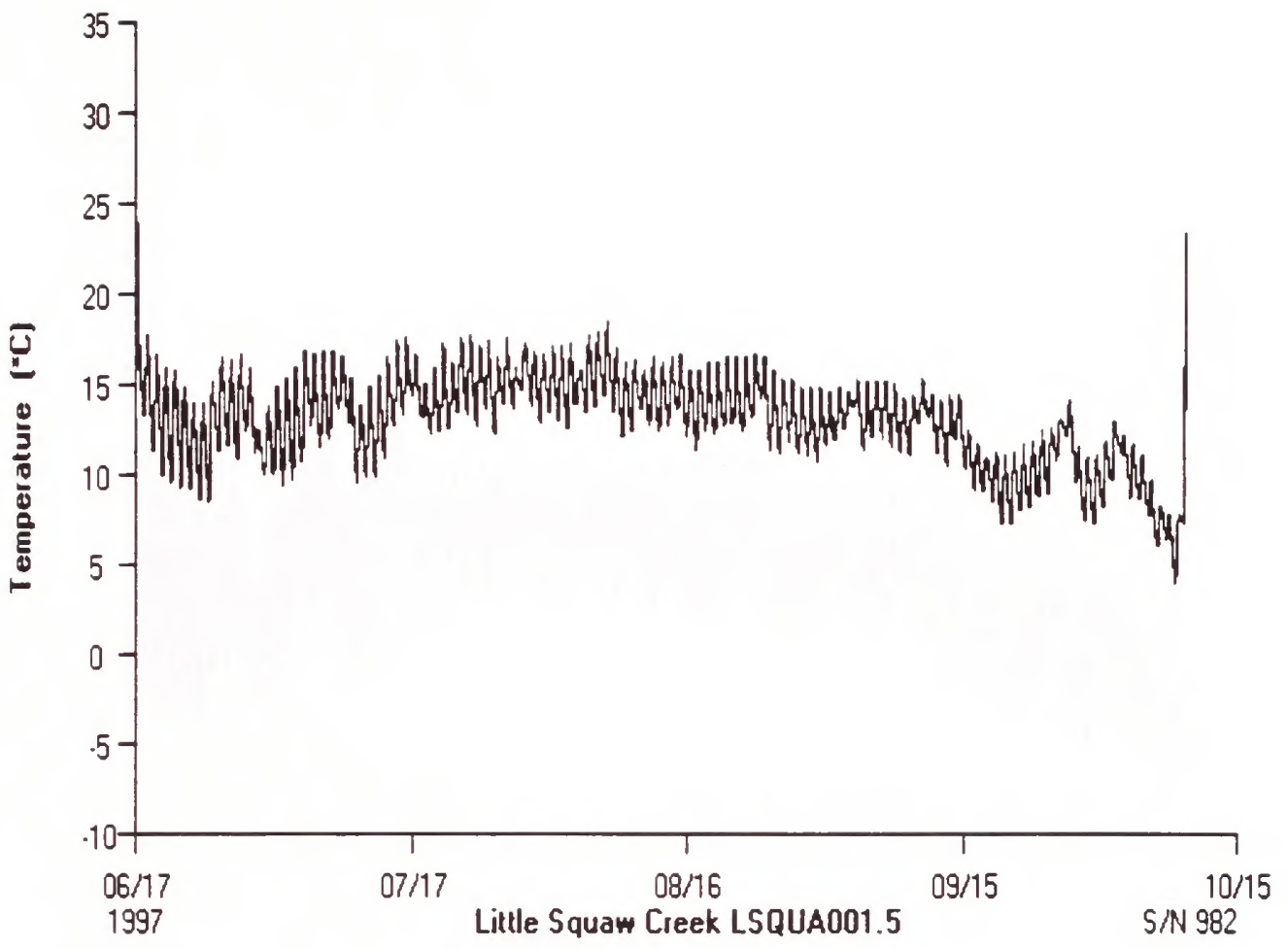

Appendix 2B.

Recording thermograph data for Little Squaw Creek, Owyhee County, ID. Site is located at Stream Mile 1.5, Legal Description T1N R5W S34 NESE, Elevation 1225m. Thermograph was set on 6/17/97 and pulled on 10/15/97. 



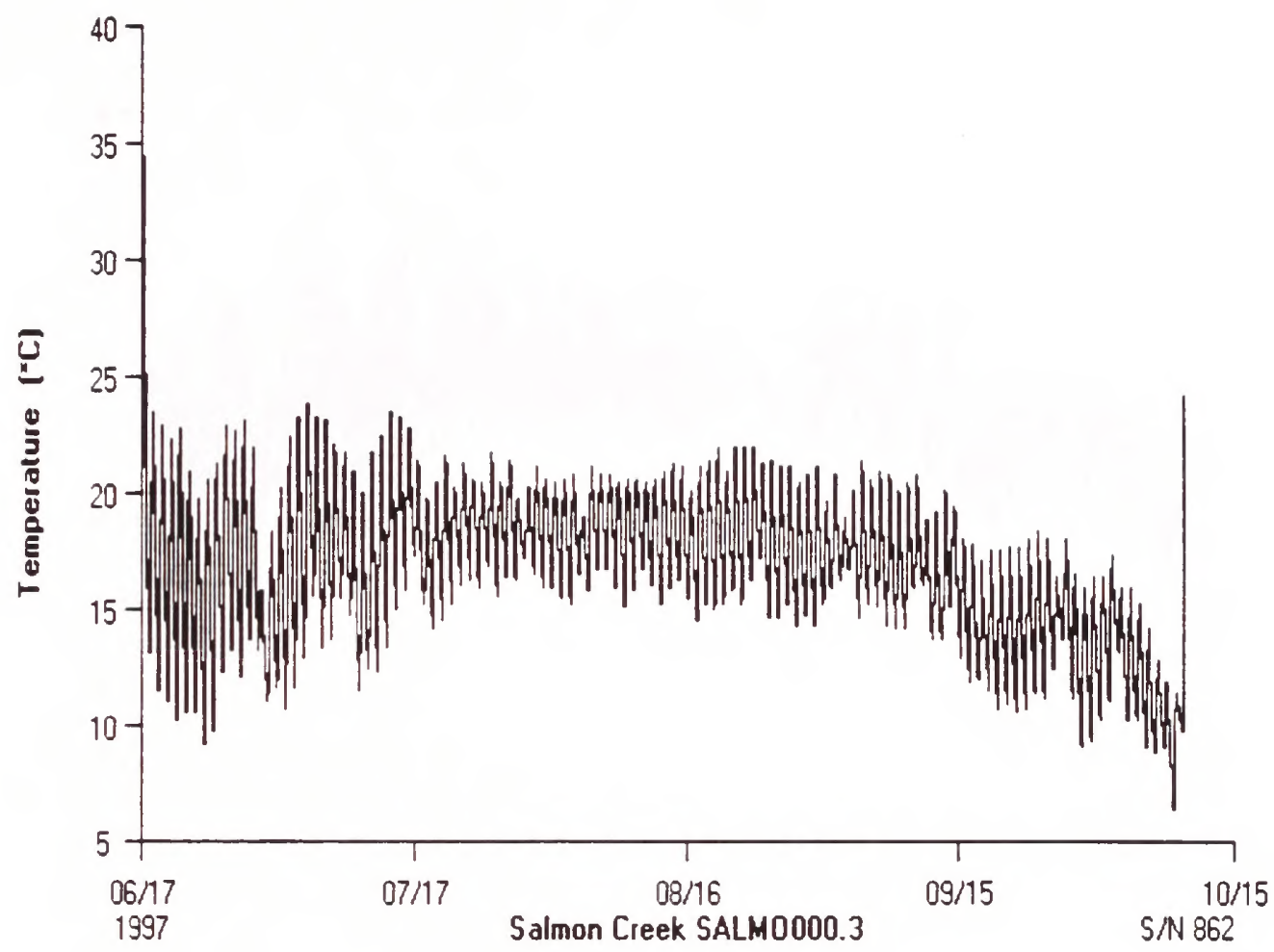

Appendix 2C.

Recording thermograph data for Salmon Creek, Owyhee County, ID. Site is located at Stream Mile 0.3, Legal Description T2S R4W S13 NWNE, Elevation 1125m. Thermograph was set on 6/17/97 and pulled on 10/15/97. 



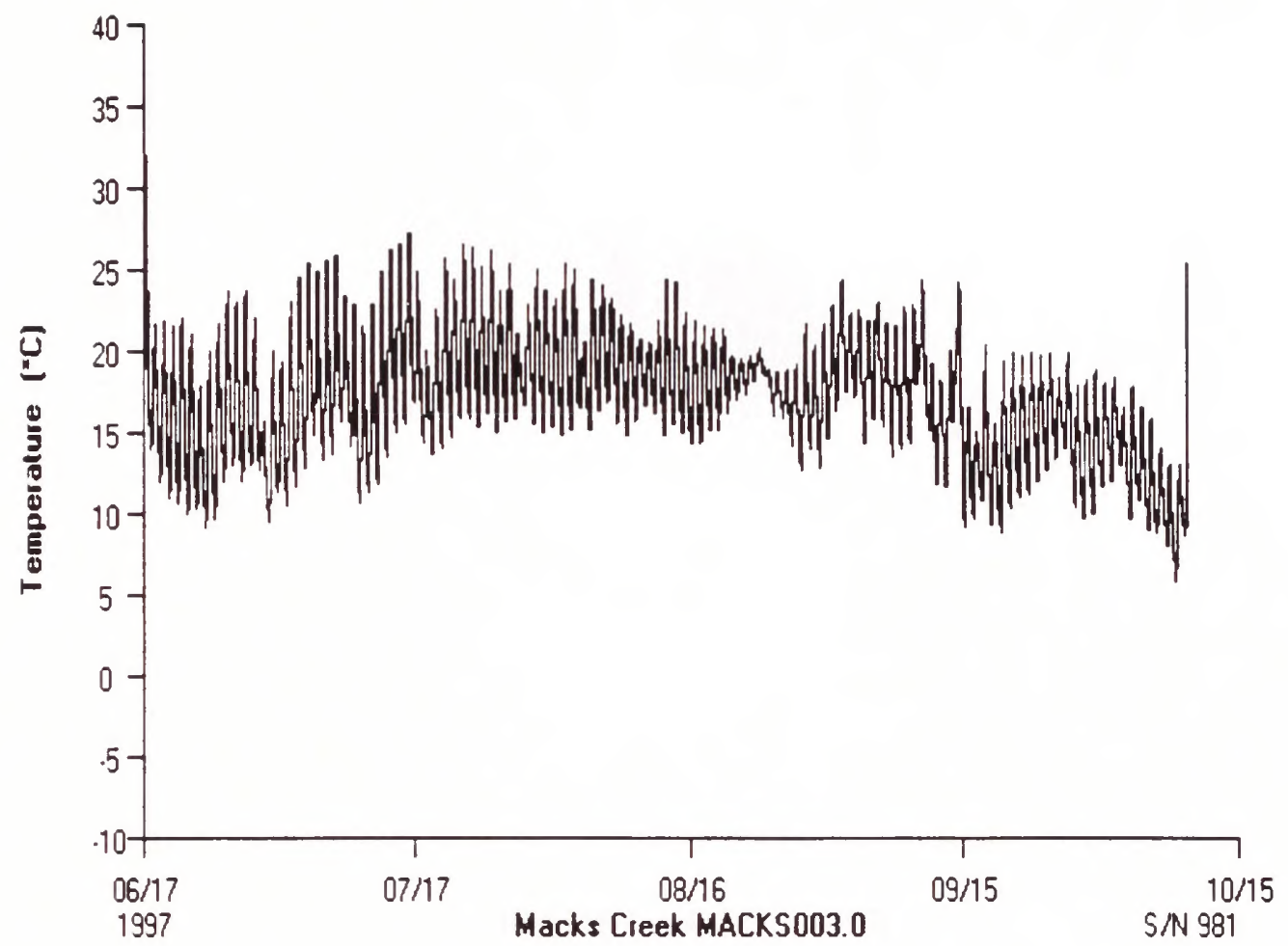

Appendix 2D.

Recording thermograph data for Macks Creek, Owyhee County, ID. Site is located at Stream Mile 3.0, Legal Description T2S R4W S27 NWNE, Elevation 1250m. Thermograph was set on 6/17/97 and pulled on 10/15/97. 



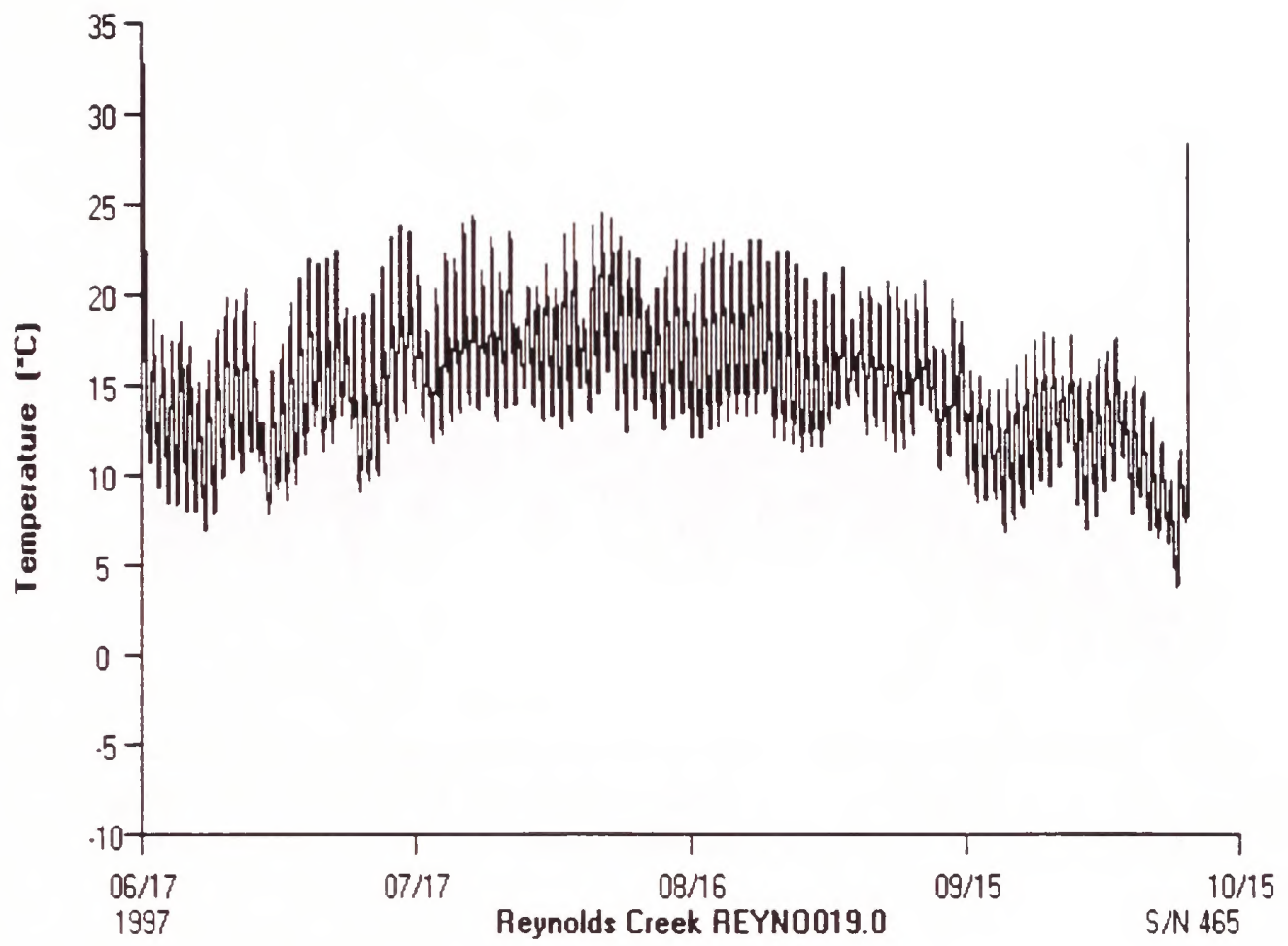

Appendix 2E.

Recording thermograph data for Reynolds Creek, Owyhee County, ID. Site is located at Stream Mile 19.0, Legal Description T3S R4W S24 NESW, Elevation 1425m. Thermograph was set on 6/17/97 and pulled on 10/15/97. 



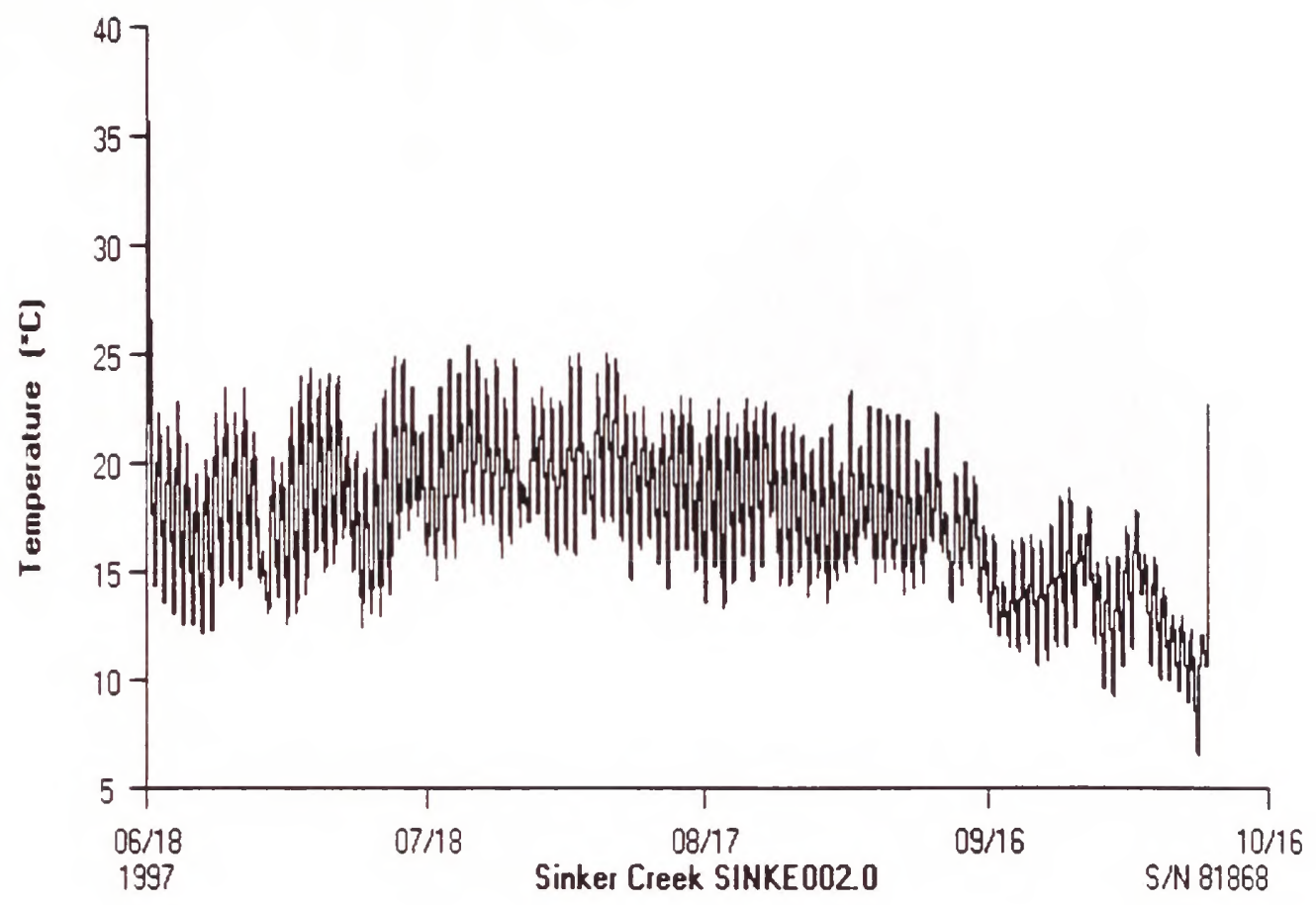

Appendix 2F.

Recording thermograph data for Sinker Creek, Owyhee County, ID. Site is located at Stream Mile 2.0, Legal Description T3S R1W S13 SWNE, Elevation 825m. Thermograph was set on 6/18/97 and pulled on 10/16/97. 



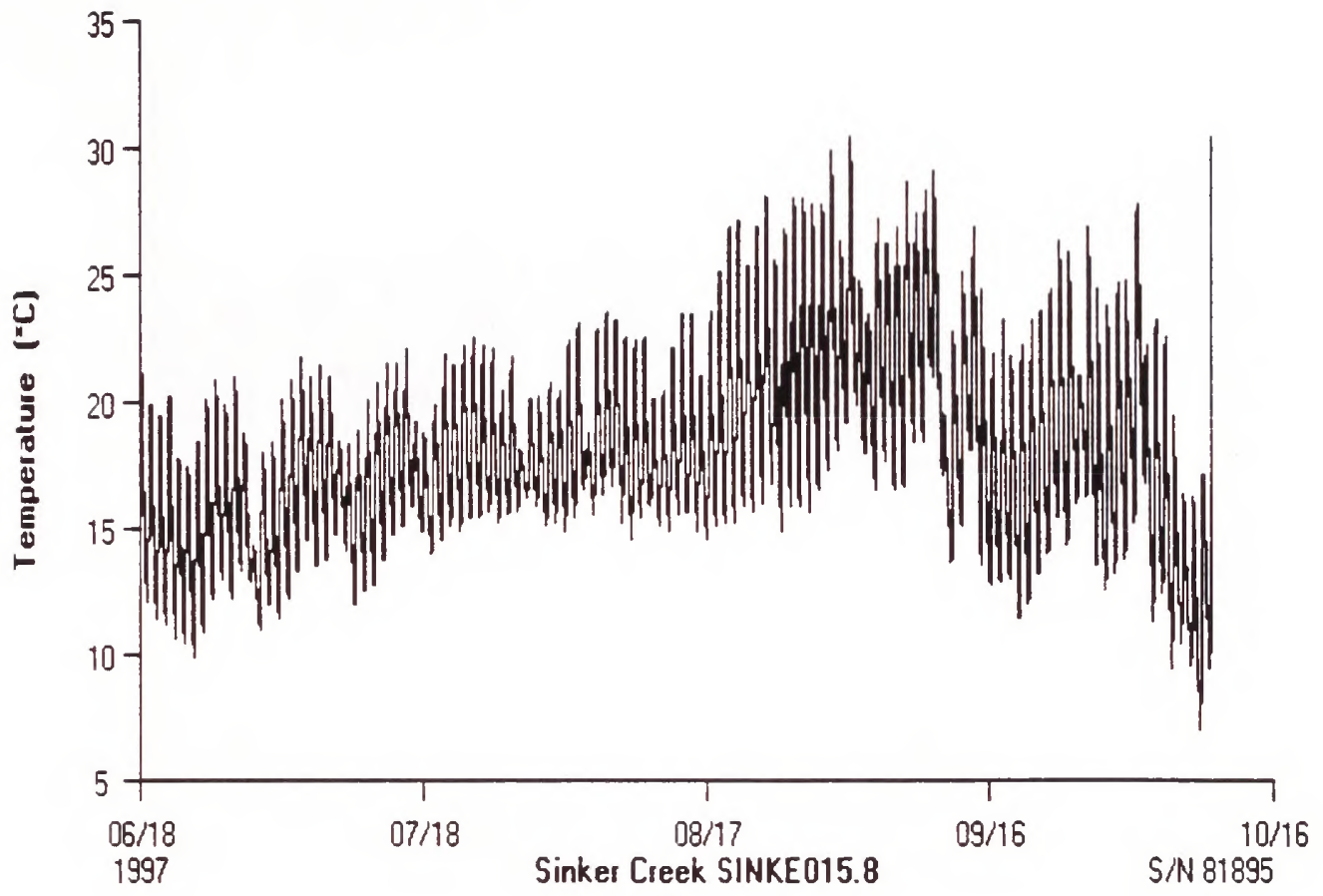

Appendix 2G.

Recording thermograph data for Sinker Creek, Owyhee County, ID. Site is located at Stream Mile 15.8, Legal Description T4S R2W S19 NENW, Elevation 1125m. Thermograph was set on 6/18/97 and pulled on 10/16/97 (Stream was dry when pulled). 



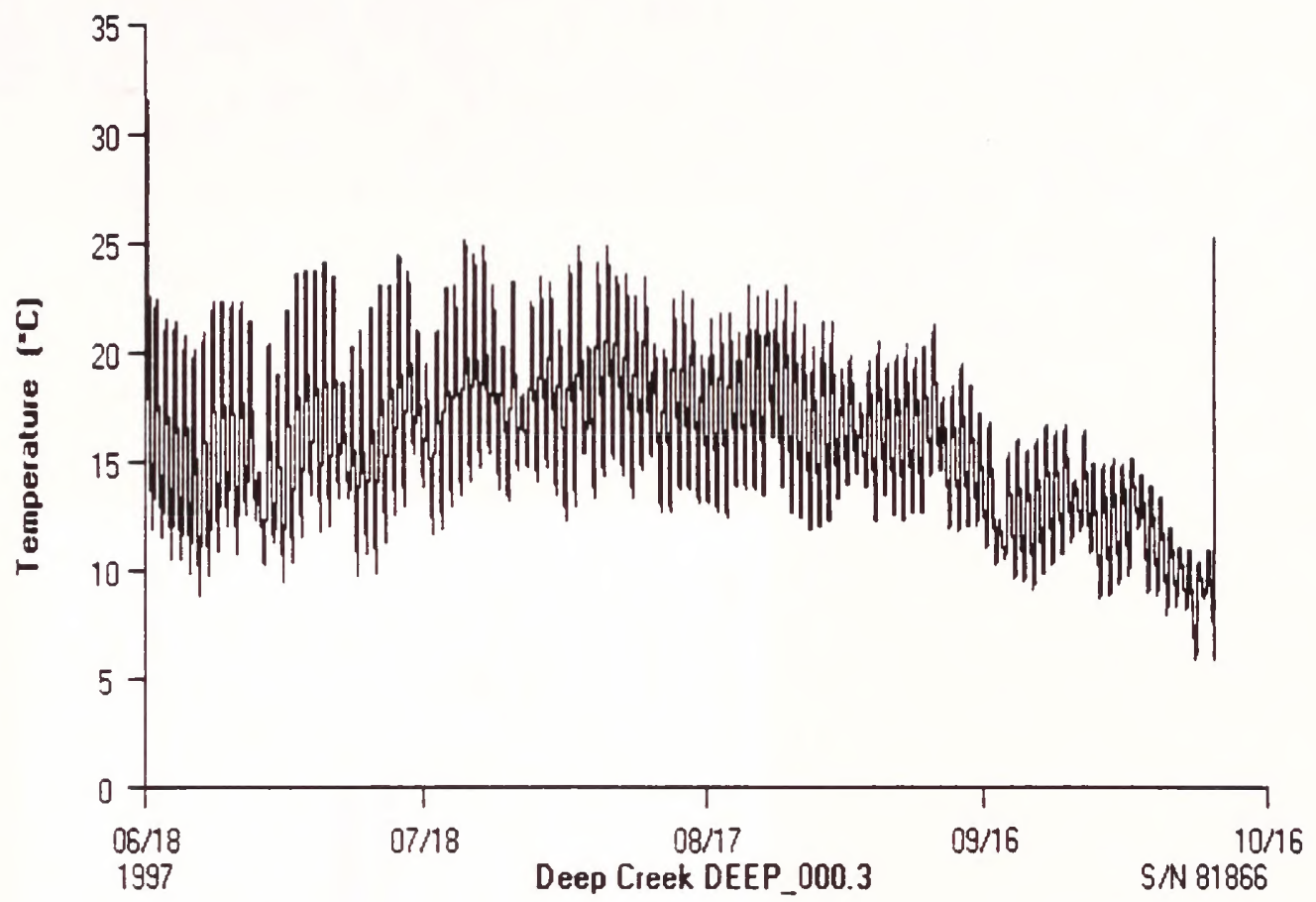

Appendix $2 \mathrm{H}$.

Recording thermograph data for Deep Creek, Owyhee County, ID. Site is located at Stream Mile 18.2, Legal Description T10S R3W S3 NWSE, Elevation $1700 \mathrm{~m}$. Thermograph was set on 6/18/97 and pulled on 10/16/97. 



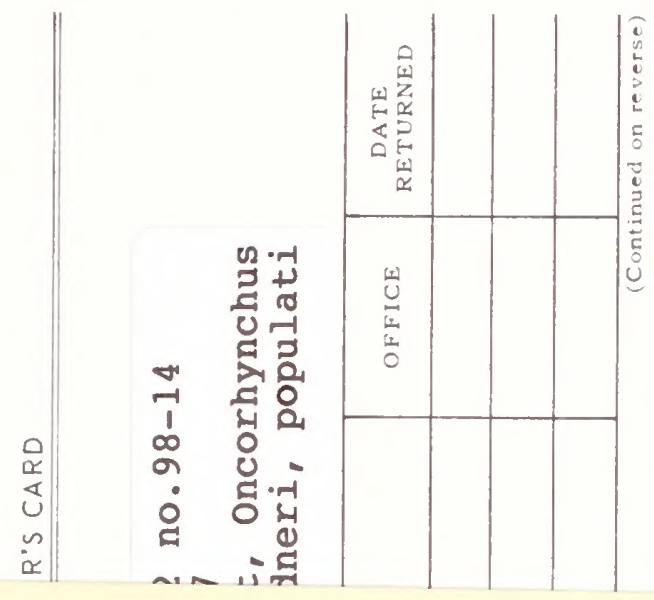

QL 84.2 .L352 no.98-14

88055557

Redband trout, Oncorhynchus mykiss gairdneri, populati

SLUU JU,

DENVER FEDERAL CENTER

P.O.BOX 25047

DENVEA, COLORADO 80225 


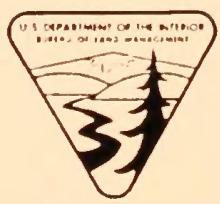

Bureau of Land Management

Idaho State Office

1387 S. Vinnell Way

Boise, Idaho 83709

BLM/ID/PT-99/002+1150 